\title{
Gravitational-wave tail effects to quartic non-linear order
}

\author{
Tanguy Marchand, ${ }^{1,2, *}$ Luc Blanchet, ${ }^{1, \dagger}$ and Guillaume Faye ${ }^{1, \ddagger}$ \\ ${ }^{1} \mathcal{G} \mathbb{R} \varepsilon \mathbb{C O}$, Institut d'Astrophysique de Paris, \\ UMR 7095, CNRS, Sorbonne Universités \& UPMC Univ Paris 6, \\ 98 bis boulevard Arago, 75014 Paris, France \\ ${ }^{2}$ Laboratoire APC - Astroparticule et Cosmologie, \\ Université Paris Diderot Paris 7, 75013 Paris, France
}

(Dated: November 29, 2016)

\begin{abstract}
Gravitational-wave tails are due to the backscattering of linear waves onto the space-time curvature generated by the total mass of the matter source. The dominant tails correspond to quadratic non-linear interactions and arise at the one-and-a-half post-Newtonian $(1.5 \mathrm{PN})$ order in the gravitational waveform. The "tails-of-tails", which are cubic non-linear effects appearing at the 3PN order in the waveform, are also known. We derive here higher non-linear tail effects, namely those associated with quartic non-linear interactions or "tails-of-tails-of-tails", which are shown to arise at the 4.5PN order. As an application, we obtain at that order the complete coefficient in the total gravitational-wave energy flux of compact binary systems moving on circular orbits. Our result perfectly agrees with black-hole perturbation calculations in the limit of extreme mass ratio of the two compact objects.
\end{abstract}

PACS numbers: 04.25.Nx, 04.30.-w, 97.60.Jd, 97.60.Lf

*Electronic address: tanguy.marchand@iap.fr

${ }^{\dagger}$ Electronic address: luc.blanchet@iap.fr

${ }_{\ddagger}^{\ddagger}$ Electronic address: guillaume.faye@iap.fr 


\section{INTRODUCTION}

The LIGO/VIRGO collaboration reported recently the observation of gravitational waves from the coalescence of black-hole binary systems [1, 2]. Both analytic works on the twobody problem in general relativity and extensive numerical relativity calculations play a very important role when interpreting and deciphering the gravitational-wave signals [3-6].

Our motivation in the present paper, is to find high-order post-Newtonian (PN) expressions of the gravitational-wave field generated by the coalescence of compact binary systems (including black-hole binaries), in the inspiralling phase preceding the final merger. Such expressions are important for the data analysis of the ground-based detectors, as well as for the future space-based eLISA detector. Moreover, they are used for high accuracy comparisons with the results of numerical relativity.

The current state of the art regarding the gravitational-wave field can be summarized by listing the various orders that have been obtained: ${ }^{1}$ namely, $3.5 \mathrm{PN}$ order for the energy flux [7-10], 3PN for the waveform and polarizations [11-13], and 3.5PN for the dominant gravitational-wave modes $[14,15]$. Regarding the equations of motion, we have now reached the $4 \mathrm{PN}$ order [16-21], while the elucidation of the previous 3PN order had took quite some time [22-32]. See [5] for a review, and also for the crucial inclusion of spin effects, both in the equations of motion and radiation field.

Our general aim is to extend the radiation field calculations up to 4.5PN order, both for the gravitational-wave energy flux (which rules the orbital phase evolution) and for the polarization waveforms and modes. The first part of this program consists in dealing with the long computations of the multipole moments of the compact binary at that order, notably the mass type quadrupole moment at the 4PN order. We shall leave these computations for future work.

The second part, addressed in this paper, consists of investigating the various non-linear interactions between those multipole moments. The most crucial contributions are due to the so-called gravitational-wave tails [33-37], which are non-linear effects (quadratic at least) physically due to the backscattering of linear waves onto the space-time curvature generated by the mass of the source. The tail effects imply a non-locality in time (i.e., a dependence on the full past history of the source) so that they may also be qualified as hereditary [37]. The tails arise dominantly at the $1.5 \mathrm{PN}$ order in the waveform, and can be directly tested by the measurement of PN parameters in LIGO/VIRGO observations [1, 2].

The tails generated by tails themselves, the so-called "tails-of-tails", come from a cubic interaction and appear dominantly at the 3PN order in the waveform [8]. Their contribution can also be potentially tested by LIGO/VIRGO observations. At the 4.5PN order, new terms called "tails-of-tails-of-tails" arise. They come from a quartic interaction between three mass monopoles $M$ (the ADM mass of the source) and the mass quadrupole moment of the source, say $M_{i j}$. The aim of the present paper is to compute this quartic multipole interaction $M \times M \times M \times M_{i j}$ in the asymptotic waveform at large distances.

Our calculation is based on the multipolar-post-Minkowskian (MPM) algorithm for generating non-linear interactions between multipole moments in the region outside a general

\footnotetext{
${ }^{1}$ As usual, $n \mathrm{PN}$ order terms mean terms going up to $\sim c^{-2 n}$ relatively to the Newtonian quadrupole formula for the wave field, and beyond the Newtonian acceleration for the equations of motion. Notice the difference of $2.5 \mathrm{PN}$ between the two nomenclatures, which originates from the fact that the "Newtonian" quadrupole formula corresponds to a $2.5 \mathrm{PN}$ radiation reaction effect in the equations of motion.
} 
isolated matter source [8, 38, 39]. Our main result is the so-called "radiative" mass-type quadrupole moment observed at large distances from that source (following the definition of [35]), given as a functional of the "source" mass-type quadrupole moment $M_{i j}$. From that result, we are able to compute the complete 4.5PN coefficient in the gravitational-wave energy flux for binaries of (non-spinning) compact objects moving on circular orbits. However, the 4PN coefficient remains unknown (except in the test mass limit). We relegate its computation to future work. The reason why we can nonetheless fully compute the 4.5PN term stems from the fact that half-integral PN approximations in the flux are only made up of hereditary tail contributions in the case of circular orbits. We will find that our 4.5PN term agrees, in the test mass limit for one of the compact bodies, with the expression derived from black-hole perturbation theory [40-49].

The plan of this paper is as follows. Sec. II is dedicated to presenting the necessary material that comes from the MPM approach to gravitational-wave generation. We investigate in Sec. III the integration formulas needed to perform the required non-linear iterations of the metric. In Sec. IV, we obtain the leading $1 / r$ part of the quartic-order metric and deduce from it the radiative quadrupole moment. Finally, in Sec. V, we derive the complete 4.5PN coefficient in the total energy flux of compact binaries for circular orbits. The paper ends with a short conclusion in Sec. VI and two technical Appendices.

\section{REVIEW ON THE MULTIPOLAR-POST-MINKOWSKIAN FORMALISM}

The gravitational field in the region outside an isolated matter source, solving the vacuum Einstein field equations in the exterior of that source, is written in "gothic" Minkowskian deviation form ${ }^{2}$ and formally developed as a post-Minkowskian or non-linearity expansion,

$$
h^{\mu \nu}=G h_{1}^{\mu \nu}+G^{2} h_{2}^{\mu \nu}+G^{3} h_{3}^{\mu \nu}+G^{4} h_{4}^{\mu \nu}+\mathcal{O}\left(G^{5}\right),
$$

where the powers of the Newton constant $G$ label the successive approximations. Then, each of the post-Minkowskian coefficients is computed as a non-linear functional of two infinite sets of time varying symmetric-trace-free (STF) multipole moments, of mass type, $M_{L}(t)$, and current type, $S_{L}(t) .{ }^{3}$ However, among these moments, the mass monopole $M$ is identified with the constant ADM mass of the source, whereas the constant current dipole $S_{i}$ coincides with the total angular momentum. The moments $M_{L}$ and $S_{L}$ specifically refer here to the so-called "canonical" multipole moments as defined in Ref. [13]. The multipolar-postMinkowskian (MPM) metric (2.1) can in principle be determined to any order by means

\footnotetext{
${ }^{2}$ We have thus $h^{\mu \nu}=\sqrt{-g} g^{\mu \nu}-\eta^{\mu \nu}$, where $\eta^{\mu \nu}$ is the (inverse) Minkowski metric, while $g$ and $g^{\mu \nu}$ are the determinant and the inverse of the covariant metric $g_{\mu \nu}$. Our signature is almost plus, i.e., $\eta_{\mu \nu}=\operatorname{diag}(-1,1,1,1)=\eta^{\mu \nu}$. We use Cartesian coordinates and solve iteratively the Einstein field equations using the harmonic coordinates condition $\partial_{\nu} h^{\mu \nu}=0$.

${ }^{3}$ Here, $L=i_{1} \cdots i_{\ell}$ denotes a multi-index composed of $\ell$ spatial indices (ranging from 1 to 3 ); we pose $L-1=i_{1} \cdots i_{\ell-1}$, and so on; $\partial_{L}=\partial_{i_{1}} \cdots \partial_{i_{\ell}}$ is the product of $\ell$ partial derivatives $\partial_{i} \equiv \partial / \partial x^{i}$; similarly, we shall write $x_{L}=x_{i_{1}} \cdots x_{i_{\ell}}$, with $x_{i}=x^{i}$ being the spatial position, and $n_{L}=n_{i_{1}} \cdots n_{i_{\ell}}$ with $n_{i}=x_{i} / r$. Symmetrization over indices is denoted by $T_{(i j)}=\frac{1}{2}\left(T_{i j}+T_{j i}\right)$. The STF projection is indicated with a hat, e.g., $\hat{n}_{L} \equiv \operatorname{STF}\left[n_{L}\right]$, or with angular brackets \langle\rangle surrounding the relevant indices, e.g., $x_{\langle i} v_{j\rangle}=$ $x_{(i} v_{j)}-\frac{1}{3} \delta_{i j} x_{k} v_{k}$. The multipole moments $M_{L}$ and $S_{L}$ are STF, e.g., $M_{L}=\hat{M}_{L}=M_{\langle L\rangle}$. Time derivatives of the moments are indicated by superscripts $(n)$. We often pose $c=1$ and $G=1$.
} 
of the iterative algorithm described in Sec. 2 of [39] (see also Sec. 2.3 in the review [5]). The resulting MPM metric represents the most general solution of the vacuum Einstein field equations outside the source in harmonic coordinates.

In the present paper, we are interested in non-linear interactions between the mass $M$ and the mass-type quadrupole moment $M_{i j}$ (having $\ell=2$ ). Accordingly, we start the iteration with a linearized metric made only of two pieces, corresponding to the contributions of $M$ and $M_{i j}$. Then, the quadratic metric involves three terms corresponding to the various possible interactions between those moments (including with themselves), and so on. With an obvious notation,

$$
\begin{aligned}
& h_{1}^{\mu \nu}=h_{M}^{\mu \nu}+h_{M_{i j}}^{\mu \nu}, \\
& h_{2}^{\mu \nu}=h_{M^{2}}^{\mu \nu}+h_{M \times M_{i j}}^{\mu \nu}+h_{M_{i j} \times M_{k l}}^{\mu \nu}, \\
& h_{3}^{\mu \nu}=h_{M^{3}}^{\mu \nu}+h_{M^{2} \times M_{i j}}^{\mu \nu}+h_{M \times M_{i j} \times M_{k l}}^{\mu \nu}+h_{M_{i j} \times M_{k l} \times M_{m n}}^{\mu \nu} .
\end{aligned}
$$

The iteration is stopped when reaching the non-linear level that is aimed for, which will here be quartic. In fact, at that level, we are interested only in the $M^{3} \times M_{i j}$ quartic interactions, involving three masses and one quadrupole moment, i.e., we look for the term

$$
h_{4}^{\mu \nu}=\cdots+h_{M^{3} \times M_{i j}}^{\mu \nu}+\cdots
$$

Using the MPM algorithm [8, 38, 39], this term $h_{M^{3} \times M_{i j}}$ in the gravitational field will be obtained by integrating the associated source term $\Lambda_{M^{3} \times M_{i j}}$ entering the vacuum Einstein field equations in harmonic coordinates. Thus, our task will amount to solving the ordinary d'Alembertian equation

$$
\square h_{M^{3} \times M_{i j}}^{\mu \nu}=\Lambda_{M^{3} \times M_{i j}}^{\mu \nu},
$$

with $\square=\eta^{\mu \nu} \partial_{\mu \nu}$, while imposing the harmonic gauge condition $\partial_{\nu} h_{M^{3} \times M_{i j}}^{\mu \nu}=0$. The source term $\Lambda_{M^{3} \times M_{i j}}$ will be a very complicated expression built from the previous iterations (2.2), see e.g., Sec. 2 of [5] for more details.

Several non-linear interactions are already known, starting with all the interactions $M \times M \times \cdots$ that involve only the mass monopole, since they simply reconstitute the Schwarzschild metric in harmonic coordinates. The quadratic piece $h_{M \times M_{i j}}$, which involves the quadratic tails, was obtained in [37]. The quadratic interaction between two quadrupole moments, namely $h_{M_{i j} \times M_{k l}}$, was derived in [39]. It contains the well known non-linear memory effect [37, 39, 50-55], as well as "semi-hereditary" contributions related to the energy and angular momentum losses by radiation [37]. The cubic piece $h_{M^{2} \times M_{i j}}$, containing the cubic "tails-of-tails", was investigated in [8]. The cubic interactions $h_{M \times M_{i j} \times M_{k l}}$ and $h_{M_{i j} \times M_{k l} \times M_{m n}}$ are not known. Finally the quartic piece $h_{M^{3} \times M_{i j}}$ we are interested in contains the "tailsof-tails-of-tails" contribution to the metric.

The mass and quadrupole parts in (2.2) are given explicitly by

$$
\begin{aligned}
h_{M}^{00} & =-4 r^{-1} M, \\
h_{M}^{0 i} & =0, \\
h_{M}^{i j} & =0,
\end{aligned}
$$


and

$$
\begin{aligned}
h_{M_{i j}}^{00} & =-2 n_{a b} r^{-3}\left[3 M_{a b}(t-r)+3 r M_{a b}^{(1)}(t-r)+r^{2} M_{a b}^{(2)}(t-r)\right], \\
h_{M_{i j}}^{0 i} & =-2 n_{a} r^{-2}\left[M_{a i}^{(1)}(t-r)+r M_{a i}^{(2)}(t-r)\right], \\
h_{M_{i j}}^{i j} & =-2 r^{-1} M_{i j}^{(2)}(t-r),
\end{aligned}
$$

where the quadrupole moment and its time derivatives depend on the retarded time $t-r$.

At the quadratic level, we have

$$
\begin{aligned}
& h_{M^{2}}^{00}=-7 r^{-2} M^{2}, \\
& h_{M^{2}}^{0 i}=0, \\
& h_{M^{2}}^{i j}=-n_{i j} r^{-2} M^{2},
\end{aligned}
$$

and

$$
\begin{array}{rl}
h_{M \times M_{i j}}^{00} & =M n_{a b} r^{-4}\left[-21 M_{a b}-21 r M_{a b}^{(1)}+7 r^{2} M_{a b}^{(2)}+10 r^{3} M_{a b}^{(3)}\right] \\
& +8 M n_{a b} \int_{1}^{+\infty} \mathrm{d} y Q_{2}(y) M_{a b}^{(4)}(t-r y) \\
h_{M \times M_{i j}}^{0 i} & =M n_{i a b} r^{-3}\left[-M_{a b}^{(1)}-r M_{a b}^{(2)}-\frac{1}{3} r^{2} M_{a b}^{(3)}\right] \\
& +M n_{a} r^{-3}\left[-5 M_{a i}^{(1)}-5 r M_{a i}^{(2)}+\frac{19}{3} r^{2} M_{a i}^{(3)}\right] \\
& +8 M n_{a} \int_{1}^{+\infty} \mathrm{d} y Q_{1}(y) M_{a i}^{(4)}(t-r y), \\
h_{M \times M_{i j}}^{i j} & M n_{i j a b} r^{-4}\left[-\frac{15}{2} M_{a b}-\frac{15}{2} r M_{a b}^{(1)}-3 r^{2} M_{a b}^{(2)}-\frac{1}{2} r^{3} M_{a b}^{(3)}\right] \\
& +M \delta_{i j} n_{a b} r^{-4}\left[-\frac{1}{2} M_{a b}-\frac{1}{2} r M_{a b}^{(1)}-2 r^{2} M_{a b}^{(2)}-\frac{11}{6} r^{3} M_{a b}^{(3)}\right] \\
& +M n_{a(i} r^{-4}\left[6 M_{j) a}+6 r M_{j) a}^{(1)}+6 r^{2} M_{j) a}^{(2)}+4 r^{3} M_{j) a}^{(3)}\right] \\
& +M r^{-4}\left[-M_{i j}-r M_{i j}^{(1)}-4 r^{2} M_{i j}^{(2)}-\frac{11}{3} r^{3} M_{i j}^{(3)}\right] \\
& +8 M \int_{1}^{+\infty} \mathrm{d} y Q_{0}(y) M_{i j}^{(4)}(t-r y) .
\end{array}
$$

In the "instantaneous" terms, the quadrupole moment is always evaluated at instant $t-r$. The tail integrals stricto sensu, which depend hereditarily on all past values of the quadrupole moment (evaluated at earlier time $t-r y$ with $y \geqslant 1$ ), also contain some specific integration kernel, which turns out to be the Legendre function of the second kind $Q_{\ell}(y)$. The most relevant form of the Legendre function for the present purpose is displayed in Eq. (3.5). See Ref. [39] for the calculation of the lengthy interactions $M_{i j} \times M_{k l}$.

At the cubic level, we have

$$
h_{M^{3}}^{00}=-8 r^{-3} M^{3}
$$




$$
\begin{aligned}
& h_{M^{3}}^{0 i}=0, \\
& h_{M^{3}}^{i j}=0,
\end{aligned}
$$

while the expressions of the tails-of-tails $M^{2} \times M_{i j}$ are provided in Ref. [8] at the leading asymptotic order in the distance to the source, when $r \rightarrow+\infty$ with $t-r$ fixed. Here however, having in view the next iteration to compute the tails-of-tails-of-tails, we shall need first to generalize the latter result to the whole space, i.e., to obtain the tails-of-tails at any distance $r$ (larger than the size of the source). Thus, it is worth supplying some more details on the calculation of tails-of-tails.

The source is the sum of a local or instantaneous part, and of an hereditary or tail part:

$$
\Lambda_{M^{2} \times M_{i j}}^{\mu \nu}=I_{M^{2} \times M_{i j}}^{\mu \nu}+T_{M^{2} \times M_{i j}}^{\mu \nu} .
$$

The hereditary part of that cubic source is merely due to the interaction between $M$ and the tail integrals present in Eqs. (2.8). We have

$$
\begin{aligned}
I_{M^{2} \times M_{i j}}^{00}= & M^{2} n_{a b} r^{-7}\left[-516 M_{a b}-516 r M_{a b}^{(1)}-304 r^{2} M_{a b}^{(2)}\right. \\
& \left.-76 r^{3} M_{a b}^{(3)}+108 r^{4} M_{a b}^{(4)}+40 r^{5} M_{a b}^{(5)}\right] \\
I_{M^{2} \times M_{i j}}^{0 i} & M^{2} \hat{n}_{i a b} r^{-6}\left[4 M_{a b}^{(1)}+4 r M_{a b}^{(2)}-16 r^{2} M_{a b}^{(3)}+\frac{4}{3} r^{3} M_{a b}^{(4)}-\frac{4}{3} r^{4} M_{a b}^{(5)}\right] \\
+ & M^{2} n_{a} r^{-6}\left[-\frac{372}{5} M_{a i}^{(1)}-\frac{372}{5} r M_{a i}^{(2)}-\frac{232}{5} r^{2} M_{a i}^{(3)}\right. \\
& \left.\quad-\frac{84}{5} r^{3} M_{a i}^{(4)}+\frac{124}{5} r^{4} M_{a i}^{(5)}\right], \\
I_{M^{2} \times M_{i j}}^{i j} & M^{2} \hat{n}_{i j a b} r^{-5}\left[-190 M_{a b}^{(2)}-118 r M_{a b}^{(3)}-\frac{92}{3} r^{2} M_{a b}^{(4)}-2 r^{3} M_{a b}^{(5)}\right] \\
+ & M^{2} \delta_{i j} n_{a b} r^{-5}\left[\frac{160}{7} M_{a b}^{(2)}+\frac{176}{7} r M_{a b}^{(3)}-\frac{596}{21} r^{2} M_{a b}^{(4)}-\frac{160}{21} r^{3} M_{a b}^{(5)}\right] \\
& +M^{2} \hat{n}_{a(i} r^{-5}\left[-\frac{312}{7} M_{j) a}^{(2)}-\frac{248}{7} r M_{j) a}^{(3)}+\frac{400}{7} r^{2} M_{j) a}^{(4)}+\frac{104}{7} r^{3} M_{j) a}^{(5)}\right] \\
& +M^{2} r^{-5}\left[-12 M_{i j}^{(2)}-\frac{196}{15} r M_{i j}^{(3)}-\frac{56}{5} r^{2} M_{i j}^{(4)}-\frac{48}{5} r^{3} M_{i j}^{(5)}\right]
\end{aligned}
$$

and

$$
\begin{aligned}
T_{M^{2} \times M_{i j}}^{00} & =M^{2} n_{a b} r^{-3} \int_{1}^{+\infty} \mathrm{d} y\left[96 Q_{0} M_{a b}^{(4)}+\left(\frac{272}{5} Q_{1}+\frac{168}{5} Q_{3}\right) r M_{a b}^{(5)}+32 Q_{2} r^{2} M_{a b}^{(6)}\right], \\
T_{M^{2} \times M_{i j}}^{0 i} & =M^{2} \hat{n}_{i a b} r^{-3} \int_{1}^{+\infty} \mathrm{d} y\left[-32 Q_{1} M_{a b}^{(4)}+\left(-\frac{32}{3} Q_{0}+\frac{8}{3} Q_{2}\right) r M_{a b}^{(5)}\right] \\
& +M^{2} n_{a} r^{-3} \int_{1}^{+\infty} \mathrm{d} y\left[\frac{96}{5} Q_{1} M_{a i}^{(4)}+\left(\frac{192}{5} Q_{0}+\frac{112}{5} Q_{2}\right) r M_{a i}^{(5)}+32 Q_{1} r^{2} M_{a i}^{(6)}\right],
\end{aligned}
$$




$$
\begin{aligned}
T_{M^{2} \times M_{i j}}^{i j} & =M^{2} \hat{n}_{i j a b} r^{-3} \int_{1}^{+\infty} \mathrm{d} y\left[-32 Q_{2} M_{a b}^{(4)}+\left(-\frac{32}{5} Q_{1}-\frac{48}{5} Q_{3}\right) r M_{a b}^{(5)}\right] \\
& +M^{2} \delta_{i j} n_{a b} r^{-3} \int_{1}^{+\infty} \mathrm{d} y\left[-\frac{32}{7} Q_{2} M_{a b}^{(4)}+\left(-\frac{208}{7} Q_{1}+\frac{24}{7} Q_{3}\right) r M_{a b}^{(5)}\right] \\
& +M^{2} \hat{n}_{a(i} r^{-3} \int_{1}^{+\infty} \mathrm{d} y\left[\frac{96}{7} Q_{2} M_{j) a}^{(4)}+\left(\frac{2112}{35} Q_{1}-\frac{192}{35} Q_{3}\right) r M_{j) a}^{(5)}\right] \\
& +M^{2} r^{-3} \int_{1}^{+\infty} \mathrm{d} y\left[\frac{32}{5} Q_{2} M_{i j}^{(4)}+\left(\frac{1536}{75} Q_{1}-\frac{96}{75} Q_{3}\right) r M_{i j}^{(5)}+32 Q_{0} r^{2} M_{i j}^{(6)}\right] .
\end{aligned}
$$

As in the tail integrals of Eqs. (2.8), in the above equations, the Legendre functions are evaluated at $y$ and the quadrupole moments are evaluated at $t-r y$.

We now apply the MPM algorithm $[8,38,39]$ to compute the $M^{2} \times M_{i j}$ metric. We first define a particular solution of the d'Alembertian equation $\square h_{M^{2} \times M_{i j}}=\Lambda_{M^{2} \times M_{i j}}$ for $\Lambda_{M^{2} \times M_{i j}}$ given by Eqs. (2.10)-(2.12), as

$$
u_{M^{2} \times M_{i j}}^{\mu \nu}=\underset{B=0}{\mathrm{FP}} \square_{R}^{-1}\left[\left(\frac{r}{r_{0}}\right)^{B} \Lambda_{M^{2} \times M_{i j}}^{\mu \nu}\right] .
$$

Here, $\square_{R}^{-1}$ denotes the usual three-dimensional retarded integral. The source term is regularized by means of a multiplying factor $\left(r / r_{0}\right)^{B}$, with $B$ being a complex number and $r_{0}$ denoting a certain constant length scale. The object (2.13) is defined by analytic continuation in $B \in \mathbb{C}$ (over the complex plane deprived of some isolated points). The finite part operation at $B=0$ (in short $\mathrm{FP}_{B=0}$ ) selects the zero-th order coefficient of the Laurent expansion when $B \rightarrow 0$. As a result of this definition, we have $\square u_{M^{2} \times M_{i j}}=\Lambda_{M^{2} \times M_{i j}}$ (so $u_{M^{2} \times M_{i j}}$ is indeed a particular solution). Moreover, $u_{M^{2} \times M_{i j}}$ has a multipolar structure similar to that of the source term.

In the next stage, we compute the divergence of (2.13), namely $w_{M^{2} \times M_{i j}}^{\mu}=\partial_{\nu} u_{M^{2} \times M_{i j}}^{\mu \nu}$. Using the fact that the divergence of the non-linear source term is zero by virtue of the Bianchi identities, i.e., $\partial_{\nu} \Lambda_{M^{2} \times M_{i j}}^{\mu \nu}=0$, which can be checked by a direct calculation on the expressions (2.11)-(2.12), we get

$$
w_{M^{2} \times M_{i j}}^{\mu}=\underset{B=0}{\mathrm{FP}} \square_{R}^{-1}\left[B\left(\frac{r}{r_{0}}\right)^{B} \frac{n^{i}}{r} \Lambda_{M^{2} \times M_{i j}}^{\mu i}\right],
$$

where the factor $B$ comes from the differentiation of the regularization factor $\left(r / r_{0}\right)^{B}$. Therefore, the solution (2.13) is not divergence-free in general but one can prove that, because of the factor $B$, its divergence is a homogeneous retarded solution of the d'Alembertian equation, i.e., $\square w_{M^{2} \times M_{i j}}^{\mu}=0$. It is then straightforward to find a correction term $v_{M^{2} \times M_{i j}}^{\mu \nu}$ satisfying at once $\square v_{M^{2} \times M_{i j}}^{\mu \nu}=0$ and $\partial_{\nu} v_{M^{2} \times M_{i j}}^{\mu \nu}=-w_{M^{2} \times M_{i j}}^{\mu}$. The equations (2.11)-(2.12) of [39] allow one to construct algorithmically $v_{M^{2} \times M_{i j}}^{\mu \nu}$ starting from $w_{M^{2} \times M_{i j}}^{\mu}$. Finally, the MPM solution of the Einstein field equations in harmonic coordinates $\left(\partial_{\nu} h_{M^{2} \times M_{i j}}^{\mu \nu}=0\right)$ reads

$$
h_{M^{2} \times M_{i j}}^{\mu \nu}=u_{M^{2} \times M_{i j}}^{\mu \nu}+v_{M^{2} \times M_{i j}}^{\mu \nu} .
$$

Following this algorithm, Ref. [8] obtained the dominant terms of the solution $h_{M^{2} \times M_{i j}}$ at infinity, when $r \rightarrow+\infty$ with $t-r=$ const. When looking only for the dominant asymptotic 
behaviour of the solution, we dispose of a simplified version of the algorithm given in the Appendix B of [8]. The result is

$$
\begin{aligned}
h_{M^{2} \times M_{i j}}^{00}= & \frac{M^{2} n_{a b}}{r} \int_{0}^{+\infty} \mathrm{d} \tau M_{a b}^{(5)}\left[-4 \ln ^{2}\left(\frac{\tau}{2 r}\right)-4 \ln \left(\frac{\tau}{2 r}\right)\right. \\
& \left.+\frac{116}{21} \ln \left(\frac{\tau}{2 r_{0}}\right)-\frac{7136}{2205}\right]+\mathcal{O}\left(\frac{1}{r^{2-\epsilon}}\right) \\
h_{M^{2} \times M_{i j}}^{0 i} & =\frac{M^{2} \hat{n}_{i a b}}{r} \int_{0}^{+\infty} \mathrm{d} \tau M_{a b}^{(5)}\left[-\frac{2}{3} \ln \left(\frac{\tau}{2 r}\right)-\frac{4}{105} \ln \left(\frac{\tau}{2 r_{0}}\right)-\frac{716}{1225}\right] \\
& +\frac{M^{2} n_{a}}{r} \int_{0}^{+\infty} \mathrm{d} \tau M_{a i}^{(5)}\left[-4 \ln ^{2}\left(\frac{\tau}{2 r}\right)-\frac{18}{5} \ln \left(\frac{\tau}{2 r}\right)\right. \\
& \left.+\frac{416}{75} \ln \left(\frac{\tau}{2 r_{0}}\right)-\frac{22724}{7875}\right]+\mathcal{O}\left(\frac{1}{r^{2-\epsilon}}\right), \\
h_{M^{2} \times M_{i j}}^{i j} & \frac{M^{2} \hat{n}_{i j a b}}{r} \int_{0}^{+\infty} \mathrm{d} \tau M_{a b}^{(5)}\left[-\ln \left(\frac{\tau}{2 r}\right)-\frac{191}{210}\right] \\
+ & \frac{M^{2} \delta_{i j} n_{a b}}{r} \int_{0}^{+\infty} \mathrm{d} \tau M_{a b}^{(5)}\left[-\frac{80}{21} \ln \left(\frac{\tau}{2 r}\right)-\frac{32}{21} \ln \left(\frac{\tau}{2 r_{0}}\right)-\frac{296}{35}\right] \\
& +\frac{M^{2} \hat{n}_{a(i}}{r} \int_{0}^{+\infty} \mathrm{d} \tau M_{j) a}^{(5)}\left[\frac{52}{7} \ln \left(\frac{\tau}{2 r}\right)+\frac{104}{35} \ln \left(\frac{\tau}{2 r_{0}}\right)+\frac{8812}{525}\right] \\
& +\frac{M^{2}}{r} \int_{0}^{+\infty} \mathrm{d} \tau M_{i j}^{(5)}\left[-4 \ln { }^{2}\left(\frac{\tau}{2 r}\right)-\frac{24}{5} \ln \left(\frac{\tau}{2 r}\right)\right. \\
& \left.+\frac{76}{15} \ln \left(\frac{\tau}{2 r_{0}}\right)-\frac{198}{35}\right]+\mathcal{O}\left(\frac{1}{r^{2-\epsilon}}\right) .
\end{aligned}
$$

The quadrupole moments in the integrands are evaluated at instant $t-r-\tau$. The notation $\mathcal{O}\left(r^{\epsilon-2}\right)$ [which could even be $o\left(r^{\epsilon-2}\right)$ ], with $0<\epsilon \ll 1$, is simply to account for the presence of logarithms of $r$ in the expansion at infinity, as the remainder is really made of a sum of some $\mathcal{O}\left(r^{-2} \ln ^{a} r\right)$, with $a=0,1$ in (2.16).

In this paper, we shall generalize Eqs. (2.16) to the whole space (the complete expressions will be too long to be displayed) and use them, along with many other interaction terms involving the lower order metrics (2.5)-(2.9), to construct the quartic source term $\Lambda_{M^{3} \times M_{i j}}$, which is precisely the source of the looked-for tails-of-tails-of-tails contribution (2.3). An important check of this calculation will be to make sure that $\partial_{\nu} \Lambda_{M^{3} \times M_{i j}}^{\mu \nu}=0$. At that stage, following again the MPM algorithm, we shall define

$$
u_{M^{3} \times M_{i j}}^{\mu \nu}=\underset{B=0}{\mathrm{FP}} \square_{R}^{-1}\left[\left(\frac{r}{r_{0}}\right)^{B} \Lambda_{M^{3} \times M_{i j}}^{\mu \nu}\right] .
$$

Since we are at the final iteration step, we shall be content with the leading asymptotic behaviour at infinity, when $r \rightarrow+\infty$ with $t-r=$ const, of the solution being constructed. There remains to compute the divergence $w_{M^{3} \times M_{i j}}$ of (2.17), to check that it is a homogeneous retarded solution of the d'Alembertian equation, at leading order in $1 / r$, and finally to add the correcting piece $v_{M^{3} \times M_{i j}}$ ensuring that the harmonic-coordinate condition is satisfied. In the large $r$ limit, Eqs. (B.4)-(B.5) of [8] are the relevant formulas to go from $w_{M^{3} \times M_{i j}}$ to $v_{M^{3} \times M_{i j}}$. In the end, our quartic metric will be built as

$$
h_{M^{3} \times M_{i j}}^{\mu \nu}=u_{M^{3} \times M_{i j}}^{\mu \nu}+v_{M^{3} \times M_{i j}}^{\mu \nu} .
$$


The leading $1 / r$ behaviour of $h_{M^{3} \times M_{i j}}$ (actually containing also $\ln ^{2} r / r$ and $\ln r / r$ terms) will be shown in Eqs. (4.1) below. The physical radiative quadrupole moment at infinity will be extracted from that metric in Eq. (4.8). As we shall see, the quartic tails-of-tailsof-tails represent dominantly a $4.5 \mathrm{PN}$ effect in the waveform, which will be specialized to compute the total energy flux generated by compact binary sources moving on circular orbits in Eq. (5.11).

\section{FORMULAS TO COMPUTE QUARTIC NON-LINEARITIES}

In this section, we present the basic integration formulas (extending notably the Appendix A of [8]) allowing for the integration of the cubic and quartic non-linearities in essentially analytic closed form. We are looking for the retarded solution of a certain d'Alembertian equation whose source term, which represents a generic term in Eqs. (2.12), is hereditary:

$$
\underset{k, m}{\Psi_{L}}=\hat{n}_{L} r^{-k} \int_{1}^{+\infty} \mathrm{d} y V_{m}(y) F(t-r y),
$$

where $\hat{n}_{L}$ is a STF product of $\ell$ unit vectors (with $L=i_{1} \cdots i_{\ell}$ ), $F(u)$ a smooth function of the retarded time that is identically zero in the remote past, i.e., $F \in \mathcal{C}^{\infty}(\mathbb{R})$ and $F(u)=0$ for $u \leqslant-\mathcal{T}$ (with $-\mathcal{T}$ being a fixed instant in the past), and where $V_{m}(y)$ is a generic function belonging to the following $m$-dependent class:

$$
\begin{aligned}
& \mathscr{V}_{m}=\left\{V(y) \in \mathcal{C}^{\infty}(] 1,+\infty[) \mid \exists a \geqslant 0, b \geqslant 0\right. \text { such that } \\
&\left.V(y) \underset{y \rightarrow+\infty}{=} \mathcal{O}\left[y^{-(m+1)} \ln ^{a}(y)\right] \text { and } V(y) \underset{y \rightarrow 1^{+}}{=} \mathcal{O}\left[\ln ^{b}(y-1)\right]\right\} .
\end{aligned}
$$

We see that the integer $m$ basically specifies the behaviour of our $\mathscr{V}_{m}$-type functions when $y \rightarrow+\infty$, while those functions are assumed to be integrable when $y \rightarrow 1^{+}$. A typical function belonging to the class $\mathscr{V}_{m}$ is the Legendre function of the second kind $Q_{m}(y)$, given by (3.5) below. Then, for $V_{m} \in \mathscr{V}_{m}$, we define the retarded multipolar solution of (3.1) as

$$
\underset{k, m}{\Psi_{L}}=\underset{B=0}{\mathrm{FP}} \square_{R}^{-1}\left[\hat{n}_{L}\left(\frac{r}{r_{0}}\right)^{B} r^{-k} \int_{1}^{+\infty} \mathrm{d} y V_{m}(y) F(t-r y)\right] .
$$

Following the prescriptions (2.13) or (2.17), this solution is defined by analytic continuation in $B \in \mathbb{C}$ as the finite part (FP) in the Laurent expansion when $B \rightarrow 0$ of the usual inverse retarded integral $\square_{R}^{-1}$ acting on the source regularized by means of the inserted factor $\left(r / r_{0}\right)^{B}$. The arbitrary constant scale $r_{0}$ will cancel out from our final physical result in Sec. V.

\section{A. Explicit closed-form representations of the solution}

We shall now present explicit forms for the general solution (3.3), i.e., analytic closedform representations for ${ }_{k, m} \Psi_{L}$. Such representations are indispensable when implementing in practice the non-linear iterative construction of the metric. In order to get the full cubic metric, whose source is the sum of (2.11) and (2.12), we need to distinguish several cases. ${ }^{4}$

\footnotetext{
${ }^{4}$ Here we do not discuss the integration of the instantaneous terms (2.11) which is comparatively much simpler than that of the hereditary terms and can be dealt with the formulas in Appendix A of [39].
} 


\section{Case where $k=1, \ell \geqslant 0$ and $m \geqslant 0$}

This case has been already investigated in Ref. [8] when $V_{m}=Q_{m}$ is the Legendre function of the second kind. The result extends naturally to all $V_{m} \in \mathscr{V}_{m}$ :

$$
\underset{1, m}{\Psi_{L}}=\hat{n}_{L} \int_{1}^{+\infty} \mathrm{d} s F^{(-1)}(t-r s)\left[Q_{\ell}(s) \int_{1}^{s} \mathrm{~d} y V_{m}(y) \frac{\mathrm{d} P_{\ell}}{\mathrm{d} y}(y)+P_{\ell}(s) \int_{s}^{+\infty} \mathrm{d} y V_{m}(y) \frac{\mathrm{d} Q_{\ell}}{\mathrm{d} y}(y)\right] .
$$

Since $F(u)$ is identically zero in the past (before some given finite instant $-\mathcal{T}$ ), we define $F^{(-1)}(u)$ to be the anti-derivative of $F$ that is also identically zero in the past. Here, $P_{\ell}(y)$ is the usual Legendre polynomial. The Legendre function $Q_{\ell}(y)$ of the second kind, with a branch cut from $y=-\infty$ to $y=1$ in the complex plane, takes the explicit form $[56]^{5}$

$$
Q_{\ell}(y)=\frac{1}{2} P_{\ell}(y) \ln \left(\frac{y+1}{y-1}\right)-\sum_{j=1}^{\ell} \frac{1}{j} P_{\ell-j}(y) P_{j-1}(y) .
$$

We recall that this function behaves as $Q_{\ell}(y) \sim y^{-\ell-1}$ when $y \rightarrow+\infty$, and that its leading expansion when $y \rightarrow 1^{+}$reads

$$
Q_{\ell}(y)=-\frac{1}{2} \ln \left(\frac{y-1}{2}\right)-H_{\ell}+\mathcal{O}(y-1)
$$

where $H_{\ell}=\sum_{j=1}^{\ell} \frac{1}{j}$ denotes the usual harmonic number.

2. Case where $k=2, \ell \geqslant 0$ and $m \geqslant 0$

For $k=2$, and still $V_{m} \in \mathscr{V}_{m}$ with $m \geqslant 0$, we start with the formula (D5) of Appendix D in Ref. [38], which yields, for the case at hands:

$$
\begin{aligned}
& \underset{2, m}{\Psi_{L}}=-\frac{\hat{n}_{L}}{2 r} \int_{-\infty}^{t-r} \mathrm{~d} \xi \int_{\frac{t-r-\xi}{2}}^{\frac{t+r+\xi}{2}} \frac{\mathrm{d} w}{w} \int_{1}^{+\infty} \mathrm{d} x V_{m}(x) F[\xi-(x-1) w] \\
& \times P_{\ell}\left[1-\frac{(t-r-\xi)(t+r-\xi-2 w)}{2 r w}\right] \text {. }
\end{aligned}
$$

Now, we define new variables $(\xi, w) \rightarrow(y, z)$ by $\xi-(x-1) w=t-r y$ and $z=1-$ $\frac{(t-r-\xi)(t+r-\xi-2 w)}{2 r w}$. With these variables we get

$$
\underset{2, m}{\Psi_{L}}=-\frac{\hat{n}_{L}}{2} \int_{1}^{+\infty} \mathrm{d} x V_{m}(x) \int_{1}^{+\infty} \mathrm{d} y F(t-r y) \int_{-1}^{1} \mathrm{~d} z \frac{P_{\ell}(z)}{\sqrt{(x y-z)^{2}-\left(x^{2}-1\right)\left(y^{2}-1\right)}} .
$$

${ }^{5}$ Two other forms useful in the present context are

$$
Q_{\ell}(y)=\frac{1}{2} \int_{-1}^{1} \mathrm{~d} x \frac{P_{\ell}(x)}{y-x}=\frac{1}{2^{\ell+1}} \int_{-1}^{1} \mathrm{~d} z \frac{\left(1-z^{2}\right)^{\ell}}{(y-z)^{\ell+1}} .
$$


By virtue of the mathematical formula (A.5) of $[8]^{6}$ we obtain:

$$
\underset{2, m}{\Psi_{L}}=-\hat{n}_{L} \int_{1}^{+\infty} \mathrm{d} s F(t-r s)\left[Q_{\ell}(s) \int_{1}^{s} \mathrm{~d} y V_{m}(y) P_{\ell}(y)+P_{\ell}(s) \int_{s}^{+\infty} \mathrm{d} y V_{m}(y) Q_{\ell}(y)\right],
$$

which has a structure similar to that of the solution (3.4).

\section{Case where $k \geqslant 2, \ell \geqslant k-2$ and $m \geqslant k-2$}

To deal with this case, it is convenient to introduce, given some positive integer $p$ and some function $V_{m} \in \mathscr{V}_{m}$, the $p$-th anti-derivative $V_{m}^{(-p)}(y)$ of $V_{m}$ that vanishes when $y=1$, together with all its derivatives of orders smaller than $p$. Namely, we define

$$
V_{m}^{(-p)}(y)=\int_{1}^{y} \mathrm{~d} x V_{m}(x) \frac{(y-x)^{p-1}}{(p-1) !}
$$

and adopt the convention that $V_{m}^{(0)}(y)=V_{m}(y)$. Such a choice is indeed meaningful for functions $V_{m}$ that satisfy the characteristic properties of the class $\mathscr{V}_{m}$. Now, for any $\ell \geqslant k-2$ and $m \geqslant k-2$, we have shown that the solution ${ }_{k, m} \Psi_{L}$ is given by

$$
\begin{aligned}
& \underset{k, m}{\Psi_{L}}=-\hat{n}_{L} \int_{1}^{+\infty} \mathrm{d} s F^{(k-2)}(t-r s)\left[Q_{\ell}(s) \int_{1}^{s} \mathrm{~d} y V_{m}^{(-k+2)}(y) P_{\ell}(y)\right. \\
& \left.+P_{\ell}(s) \int_{s}^{+\infty} \mathrm{d} y V_{m}^{(-k+2)}(y) Q_{\ell}(y)\right]
\end{aligned}
$$

which appears to be an interesting generalization of Eq. (3.9) corresponding to the case $k=2$. Notice, however, that the latter formula (3.11) is not valid in the case $k=1$. This case has to be treated separately using the result (3.4). The proof of Eq. (3.11) goes by induction on the integer $k \geqslant 2$, and is relegated to Appendix A 1 .

Stricto sensu, we are not allowed to use Eq. (3.11) when $m=0, k=3, \ell=2$, which corresponds to one of the hereditary terms of the cubic source (2.12). However, it happens to be valid also in this case. Indeed, the proof leading to Eq. (3.9) still holds for $m=-1$ as all integrals are convergent. Then, to derive the formula for $m=0, k=3, \ell=2$, we proceed similarly to the recursion presented in Appendix A 1, by performing an integration by parts and choosing for $V_{0}^{(-1)}(y)$ the anti-derivative that vanishes for $y=1$ [see Eq. (A3)].

\section{4. $\quad$ Case $\ell=0, k \geqslant 3$ and $m \geqslant k-2$}

As it turned out, one (and only one) term of the cubic source given by Eq. (2.12) does not fall into the previous cases. For this term, corresponding to the values $\ell=0, k=3$ and

6 Namely,

$$
\frac{1}{2} \int_{-1}^{1} \frac{\mathrm{d} z P_{\ell}(z)}{\sqrt{(x y-z)^{2}-\left(x^{2}-1\right)\left(y^{2}-1\right)}}=\left\{\begin{array}{l}
P_{\ell}(x) Q_{\ell}(y) \text { when } 1<x \leqslant y, \\
P_{\ell}(y) Q_{\ell}(x) \text { when } 1<y \leqslant x .
\end{array}\right.
$$


$m=2$, we need to find another formula. Fortunately, this can be done by noticing that, when $k=2$, Eq. (3.9) is true for $\ell=0$, and when $k=3$, most of the reasonings of the proof of Eq. (3.11) remain valid. More details are given in Appendix A 2. In the end, for $k=3$ we find

$$
\begin{aligned}
\underset{3, m}{\Psi_{L=0}=} & -\left[\ln \left(\frac{r}{r_{0}}\right)+1\right] \frac{F(t-r)}{r} \int_{1}^{+\infty} \mathrm{d} y V_{m}(y) \\
& +\int_{1}^{+\infty} \mathrm{d} s F^{(1)}(t-r s)\left(Q_{0}(s) \int_{1}^{s} \mathrm{~d} y(y+1) V_{m}(y)\right. \\
& \left.+\int_{s}^{+\infty} \mathrm{d} y\left[(y+1) Q_{0}(y)+\ln \left(\frac{y-1}{s-1}\right)\right] V_{m}(y)\right)
\end{aligned}
$$

where we recall that $Q_{0}(y)=\frac{1}{2} \ln \left(\frac{y+1}{y-1}\right)$. Observe the first appearance of the logarithm of $r$, in the first term of Eq. (3.12), due to the presence of a pole in the original integral when $B \rightarrow 0$. As a result the formula (3.12) explicitly depends on the arbitrary scale $r_{0}$. It will be interesting to study later the fate of such scale which must disappear from physical results. With Eq. (3.12), we have in hands enough material to integrate explicitly all the cubic hereditary source terms given by (2.12) — the integration of the instantaneous source terms (2.11) being the same as for lower orders.

We have also derived a more general formula, valid for $\ell=0, k \geqslant 3$ and $m \geqslant k-2$ :

$$
\begin{aligned}
& \underset{k, m}{\Psi_{L=0}}=\frac{(-)^{k}}{(k-2) !}\left\{\left[\ln \left(\frac{r}{r_{0}}\right)+H_{k-2}\right] \frac{F^{(k-3)}(t-r)}{r} \int_{1}^{+\infty} \mathrm{d} y V_{m}(y) \varphi_{k-2}(y)\right. \\
& -\int_{1}^{+\infty} \mathrm{d} s F^{(k-2)}(t-r s)\left(Q_{0}(s) \int_{1}^{s} \mathrm{~d} y V_{m}(y)(y+1)^{k-2}\right. \\
& \left.+\int_{s}^{+\infty} \mathrm{d} y V_{m}(y)\left[(y+1)^{k-2} Q_{0}(y)+\varphi_{k-2}(y) \ln \left(\frac{y-1}{s-1}\right)\right]\right) \\
& \left.-\sum_{i=1}^{k-3}(-)^{k+i} \frac{(k-3-i) !}{r^{k-1-i}} \int_{1}^{+\infty} \mathrm{d} y V_{m}(y) \varphi_{i}(y) F^{(i-1)}(t-r y)\right\} \text {, }
\end{aligned}
$$

where we have posed $\varphi_{i}(y)=\frac{1}{2}\left[(y+1)^{i}-(y-1)^{i}\right]$, to ease the notation, and $H_{k-2}=\sum_{j=1}^{k-2} \frac{1}{j}$. Notice the last term in (3.13), which is absent from Eq. (3.12) and constitutes an additional contribution here for $k \geqslant 4$. The proofs of (3.12) and (3.13) are presented in Appendix A 2.

For all the previous formulas in this section, we have verified explicitly that the original d'Alembertian equation (3.1) is satisfied and that the leading asymptotic behaviour, for $r \rightarrow \infty$ with $t-r$ constant, is in complete agreement with Eqs. (A.13) and (A.19) of Ref. [8] in the particular case where $V_{m}(y)=Q_{m}(y)$.

\section{B. Asymptotic expansion at future null infinity}

We now present other formulas, going beyond those investigated in Ref. [8], for studying the leading order in the asymptotic expansion when $r \rightarrow+\infty$ with $t-r$ constant, and which will enable us to control the asymptotic behaviour of the metric in the last stage of our iteration, at the quartic level. 


\section{Case $k=1, m \geqslant 0$ and $\ell \geqslant 0$}

From the result (3.4) it is straightforward to see (cf Eq. (A.7) of [8]) that, to leading order at future null infinity $(r \rightarrow+\infty$ with $t-r=$ const):

$$
\underset{1, m}{\Psi_{L}}=\frac{\hat{n}_{L}}{r} \int_{0}^{+\infty} \mathrm{d} \tau F^{(-1)}(t-r-\tau) \int_{1+\tau / r}^{+\infty} \mathrm{d} x V_{m}(x) \frac{\mathrm{d} Q_{\ell}}{\mathrm{d} x}(x)+\mathcal{O}\left(\frac{1}{r^{2-\epsilon}}\right) .
$$

We remind that the neglected terms in $\mathcal{O}\left(r^{\epsilon-2}\right)$ also include possible powers of the logarithm of $r$.

\section{Case $k \geqslant 2, \ell \geqslant k-2, m \geqslant 0$}

The formulas (A.10)-(A.17) of [8] can be extended to any function $V_{m} \in \mathscr{V}_{m}$ by means of the same procedure that was used to get them in [8]. We find in that case that

$$
\underset{k, m}{\Psi_{L}}=-\underset{k, m}{\alpha} \frac{\hat{n}_{L}}{r} F^{(k-3)}(t-r)+\mathcal{O}\left(\frac{1}{r^{2}}\right) .
$$

The coefficients are given by the following explicit although involved expressions:

$$
\begin{aligned}
\alpha_{\ell} & =\sum_{i=0}^{k-2} C_{\ell, i}^{k-2} \int_{1}^{+\infty} \mathrm{d} y V_{m}(y) Q_{\ell-k+2+2 i}(y), \\
\text { where } \quad C_{\ell, i}^{k-2} & =(-)^{i}\left(\begin{array}{c}
k-2 \\
i
\end{array}\right) \frac{(2 \ell-2 k+3+2 i) ! !}{(2 \ell+1+2 i) ! !}(2 \ell-2 k+5+4 i),
\end{aligned}
$$

with $\left(\begin{array}{c}k-2 \\ i\end{array}\right)$ denoting the usual binomial coefficient. One can check that the remaining integral is convergent for any $V_{m} \in \mathscr{V}_{m}$ as long as $\ell \geqslant k-2$, since $Q_{\ell}(y) \sim y^{-\ell-1}$ when $y \rightarrow+\infty$. Interestingly, the expression (3.16a) for the ${ }_{k, m} \alpha_{\ell}$ 's may be recast into the more compact form

$$
\underset{k, m}{\alpha}=(-)^{k} \int_{1}^{+\infty} \mathrm{d} y V_{m}(y) Q_{\ell}^{(-k+2)}(y)
$$

where $Q_{\ell}^{(-k+2)}(y)$ is the $(k-2)$-th anti-derivative of $Q_{\ell}(y)$ that vanishes at $y=+\infty$ with all its derivatives, i.e.,

$$
Q_{\ell}^{(-k+2)}(y)=-\int_{y}^{+\infty} \mathrm{d} z Q_{\ell}(z) \frac{(y-z)^{k-3}}{(k-3) !}
$$

for $k \geqslant 3$, and $Q_{\ell}^{(0)}(y) \equiv Q_{\ell}(y)$.

\section{Case $k \geqslant \ell+3$ and $m \geqslant k-\ell-2$}

Adapting the equations (A.19)-(A.21) from [8] we readily get

$$
\underset{k, m}{\Psi_{L}}=-\frac{\hat{n}_{L}}{r} \int_{0}^{+\infty} \mathrm{d} \tau F^{(k-2)}(t-r-\tau)\left[\underset{k, m}{\beta_{\ell}} \ln \left(\frac{\tau}{2 r_{0}}\right)+\underset{k, m}{\gamma_{\ell}}\right]+\mathcal{O}\left(\frac{1}{r^{2}}\right),
$$


with the explicit coefficients

$$
\begin{aligned}
\underset{k, m}{\beta_{\ell}} & =\frac{1}{2} \int_{1}^{+\infty} \mathrm{d} x V_{m}(x) \int_{-1}^{1} \mathrm{~d} z \frac{(z-x)^{k-3}}{(k-3) !} P_{\ell}(z), \\
\underset{k, m}{\gamma_{\ell}} & =\frac{1}{2} \int_{1}^{+\infty} \mathrm{d} x V_{m}(x) \int_{-1}^{1} \mathrm{~d} z \frac{(z-x)^{k-3}}{(k-3) !} P_{\ell}(z)\left[-\ln \left(\frac{x-z}{2}\right)+H_{k-3}\right] .
\end{aligned}
$$

\section{Case $k=4, \ell=0$ and $m=0$}

So far, we have just extended in a natural way the integration formulas of [8] (see also the Appendix A of [15] for other formulas). However, in our computation, one extra case must still be dealt with, corresponding to the values $k=4, \ell=0$ and $m=0$. Because $m$ vanishes, the function $V_{0} \in \mathscr{V}_{0}$ does not go to zero fast enough when $y \rightarrow+\infty$ to ensure the convergence of the coefficients (3.20). To handle that case we use the lemma 7.2 of Ref. [38]. Following the same notation, we define by analytic continuation the $B$-dependent function

$$
R_{B}(r, s)=\frac{1}{2 r_{0}^{B}} \int_{0}^{r} \mathrm{~d} x x^{B-3} \int_{1}^{+\infty} \mathrm{d} y V_{0}(y) F[s-x(y-1)] .
$$

We can then write the leading term of the asymptotic expansion of the solution at infinity, for any $V_{0} \in \mathscr{V}_{0}$, as

$$
\underset{4,0}{\Psi_{L=0}}=\frac{1}{r} \underset{B=0}{\mathrm{FP}} \int_{-\infty}^{t-r} \mathrm{~d} s R_{B}\left(\frac{t-r-s}{2}, s\right)+\mathcal{O}\left(\frac{1}{r^{2-\epsilon}}\right)
$$

Inserting (3.21) into (3.22) we get

$$
\underset{4,0}{\Psi_{L=0}}=\frac{1}{2 r} \underset{B=0}{\mathrm{FP}} \int_{-\infty}^{t-r} \mathrm{~d} s \int_{0}^{\frac{t-r-s}{2}} \mathrm{~d} x \frac{x^{B-3}}{r_{0}^{B}} \int_{1}^{+\infty} \mathrm{d} y V_{0}(y) F[s-x(y-1)]+\mathcal{O}\left(\frac{1}{r^{2-\epsilon}}\right)
$$

After the convenient change of variable $s \rightarrow z=\frac{t-r-s}{2}$, we can integrate explicitly over $z$. Furthermore, we integrate three times by part the remaining integral over $x$ so as to make the pole $\propto 1 / B$ appear. Those operations result in

$$
\begin{aligned}
\underset{4,0}{\Psi_{L=0}} & =\underset{B=0}{\operatorname{FP}}\left\{\frac{1}{2 B(B-1)(B-2)}\left(\frac{r}{r_{0}}\right)^{B} \int_{1}^{+\infty} \mathrm{d} s F^{(2)}(t-r s)(s-1)^{B} \int_{1}^{+\infty} \mathrm{d} y \frac{V_{0}(y)}{(y+1)^{B-2}}\right\} \\
& +\mathcal{O}\left(\frac{1}{r^{2-\epsilon}}\right) .
\end{aligned}
$$

The above equation enables us to integrate all the terms that are not covered by the previous formulas. Notice that the integral

$$
I_{0}(B)=\int_{1}^{+\infty} \mathrm{d} y \frac{V_{0}(y)}{(y+1)^{B-2}}
$$

in (3.24) diverges when $B=0$, since the function $V_{0}(y)$ only behaves like $y^{-1}$ when $y \rightarrow+\infty$. However, this divergence is "protected" by the analytic continuation in $B$ and it is even possible to perform a Laurent expansion of $I_{0}(B)$ as $B$ goes to zero. To this aim, let us 
consider the expansion of $V_{0}(y)$ in powers of the variable $y+1$ at infinity. For the actual source we are interested in, it turns out that

$$
V_{0}(y)=\frac{V_{-1}}{y+1}+\frac{V_{-2}}{(y+1)^{2}}+\frac{V_{-3}}{(y+1)^{3}}+\frac{V_{-3}^{\log } \ln (y+1)}{(y+1)^{3}}+\delta V_{-4}(y),
$$

where $V_{-1}, V_{-2}, V_{-3}$ and $V_{-3}^{\log }$ are numerical constants, whereas the function $\delta V_{-4}(y)$ behaves like some power of $\ln (y-1)$ near $y=1$ and is $o\left(1 / y^{3}\right)$ near $y \rightarrow+\infty$. Thus, we have at first order in $B$ :

$$
\int_{1}^{+\infty} \mathrm{d} y \frac{\delta V_{-4}(y)}{(y+1)^{B-2}}=\int_{1}^{+\infty} \mathrm{d} y(y+1)^{2} \delta V_{-4}(y)[1-B \ln (y+1)]+\mathcal{O}\left(B^{2}\right) .
$$

Substituting to $V_{0}(y)$ its expansion (3.26) in Eq. (3.25), we find

$$
\begin{aligned}
I_{0}(B) & =\frac{2^{2-B} V_{-1}}{B-2}+\frac{2^{1-B} V_{-2}}{B-1}+\frac{2^{-B} V_{-3}}{B}+2^{-B} V_{-3}^{\log }\left(\frac{\ln 2}{B}+\frac{1}{B^{2}}\right) \\
& +\int_{1}^{+\infty} \mathrm{d} y(y+1)^{2} \delta V_{-4}(y)[1-B \ln (y+1)]+\mathcal{O}\left(B^{2}\right) .
\end{aligned}
$$

Each time that we have to apply Eq. (3.24), we use the above truncated expression for the integral $I_{0}(B)$, which is readily expanded up to the first order in $B$, and take the finite part when $B=0$ as defined in (3.24).

\section{Integrating the instantaneous logarithmic terms}

Finally, the quartic source also contains terms that are instantaneous, and thus simpler than the previous hereditary terms, but involve the logarithm of $r$. These instantaneous logarithmic terms are not covered by the solution (3.3). The problem amounts to finding an explicit representation of

$$
\begin{aligned}
\underset{k}{\chi_{L}} & =\underset{B=0}{\operatorname{FP}} \square_{R}^{-1}\left[\hat{n}_{L}\left(\frac{r}{r_{0}}\right)^{B} \ln \left(\frac{r}{r_{0}}\right) r^{-k} F(t-r)\right] \\
& =\underset{B=0}{\operatorname{FP}} \frac{\mathrm{d}}{\mathrm{d} B}\left\{\square_{R}^{-1}\left[\hat{n}_{L}\left(\frac{r}{r_{0}}\right)^{B} r^{-k} F(t-r)\right]\right\} .
\end{aligned}
$$

Notice that for all those terms the scale $r_{0}$ entering the instantaneous logarithms $\ln \left(r / r_{0}\right)$ is the same as the one of our MPM algorithm.

\section{Case $k=2$}

According to Eq. (A.2) of Ref. [39] we have (for any $B \in \mathbb{C}$ ):

$$
\square_{R}^{-1}\left[\hat{n}_{L}\left(\frac{r}{r_{0}}\right)^{B} r^{-2} F(t-r)\right]=\frac{1}{K_{\ell}(B)} \int_{r}^{+\infty} \mathrm{d} s F(t-s) \hat{\partial}_{L}\left[\frac{(s-r)^{B+\ell}-(s+r)^{B+\ell}}{r}\right],
$$


with $K_{\ell}(B)=2\left(2 r_{0}\right)^{B} B(B-1) \cdots(B-\ell)$; the operator $\hat{\partial}_{L}$ denotes a STF product of $\ell$ spatial derivatives $\left(L=i_{1} \cdots i_{\ell}\right)$. We inject (3.30) into (3.29), apply the differentiation with respect to $B$, perform the Laurent expansion when $B \rightarrow 0$, and look for the finite part coefficient. This leads to ${ }^{7}$

$$
\underset{2}{\chi_{L}}=\frac{(-)^{\ell}}{4 \ell !} \int_{r}^{+\infty} \mathrm{d} s F(t-s) \hat{\partial}_{L}\left[\frac{(s-r)^{\ell}\left(\ln \left(\frac{s-r}{2 r_{0}}\right)+H_{\ell}\right)^{2}-(s+r)^{\ell}\left(\ln \left(\frac{s+r}{2 r_{0}}\right)+H_{\ell}\right)^{2}}{r}\right],
$$

where $H_{\ell}=\sum_{j=1}^{\ell} \frac{1}{j}$ is the $\ell$-th harmonic number. An alternative, simpler representation of the right-hand side of Eq. (3.31) involving the Legendre function reads

$$
\chi_{L}=-\frac{\hat{n}_{L}}{2 r} \int_{r}^{+\infty} \mathrm{d} s F(t-s) Q_{\ell}\left(\frac{s}{r}\right)\left[\ln \left(\frac{s^{2}-r^{2}}{4 r_{0}^{2}}\right)+2 H_{\ell}\right] .
$$

To prove it, we have verified that the above function satisfies the requested d'Alembertian equation and has the same leading behaviour at infinity as the expression (3.31) of $\chi_{2 L}$. As a result, the $1 / r$ coefficient when $r \rightarrow+\infty$ with $t-r$ constant can be computed either from (3.31) using the formulas (A.35) in [38], or more directly from (3.32), by inserting the expansion (3.6) of the Legendre function $Q_{\ell}(y)$ when $y \rightarrow 1^{+}$. We get

$$
\underset{2}{\chi_{L}}=\frac{\hat{n}_{L}}{4 r} \int_{0}^{+\infty} \mathrm{d} \tau F(t-r-\tau)\left[\left(\ln \left(\frac{\tau}{2 r_{0}}\right)+2 H_{\ell}\right)^{2}-\ln ^{2}\left(\frac{r}{r_{0}}\right)\right]+\mathcal{O}\left(\frac{1}{r^{2-\epsilon}}\right) .
$$

\section{Case $3 \leqslant k \leqslant \ell+2$}

According to Eq. (A9) of [39] we have in this case:

$$
\begin{aligned}
\square_{R}^{-1}\left[\hat{n}_{L}\left(\frac{r}{r_{0}}\right)^{B} r^{-k} F(t-r)\right] & =\left(\frac{r}{r_{0}}\right)^{B} \sum_{i=0}^{k-3} \alpha_{i}(B) \hat{n}_{L} \frac{F^{(i)}(t-r)}{r^{k-i-2}} \\
& +\beta(B) \square_{R}^{-1}\left[\hat{n}_{L}\left(\frac{r}{r_{0}}\right)^{B} r^{-2} F^{(k-2)}(t-r)\right] .
\end{aligned}
$$

The $B$-dependent coefficients $\alpha_{i}(B)$ and $\beta(B)$ are given by Eqs. (A.10) of [39]. However, for $3 \leqslant k \leqslant l+2, \alpha_{i}(B)$ does not have any pole when $B \rightarrow 0$, and the expansion of $\beta(B)$ starts at the first order in $B$, i.e., $\beta(B)=B+\mathcal{O}\left(B^{2}\right)$. As the retarded integral of a source term whose radial dependence is $r^{-2}$ (with any power of the logarithm of $r$ ) does not have any pole either, we find

$$
\underset{k}{\chi_{L}}=\frac{1}{r}\left[\alpha_{k-3}(0) \ln \left(\frac{r}{r_{0}}\right)+\alpha_{k-3}^{\prime}(0)\right] \hat{n}_{L} F^{(k-3)}(t-r)
$$

\footnotetext{
${ }^{7}$ In this derivation we use the fact that, for any integer such that $0 \leqslant i \leqslant 2 \ell,[38]$

$$
\hat{\partial}_{L}\left[\frac{(s-r)^{i}-(s+r)^{i}}{r}\right]=0 .
$$
}




$$
+\beta^{\prime}(0) \underset{B=0}{\operatorname{FP}} \square_{R}^{-1}\left[\hat{n}_{L}\left(\frac{r}{r_{0}}\right)^{B} r^{-2} F^{(k-2)}(t-r)\right]+\mathcal{O}\left(\frac{1}{r^{2-\epsilon}}\right),
$$

where $\alpha_{k-3}^{\prime}(0)$ and $\beta^{\prime}(0)$ denote the $B$-derivative, evaluated at $B=0$, of the coefficients $\alpha_{k-3}(B)$ and $\beta(B)$ displayed explicitly in Ref. [39]. For completeness, let us point out that

$$
\begin{aligned}
\alpha_{k-3}(0) & =-\frac{2^{k-3}(k-3) !(\ell-k+2) !}{(k-3-\ell) !(k-2+\ell) !} \\
\alpha_{k-3}^{\prime}(0) & =\alpha_{k-3}(0)\left[H_{k+\ell-2}-H_{k-3}-2 H_{\ell}+H_{\ell-k+2}\right],
\end{aligned}
$$

with $\beta(0)=0$ and $\beta^{\prime}(0)=2 \alpha_{k-3}(0)$. The equation (3.35) is sufficient for our purposes as we can compute the last term thanks to the identity (3.30).

\section{Case $k \geqslant \ell+3$}

In that case, Eq. (3.34) is still valid, but $\alpha_{k-3}(B)$ has now a simple pole while $\beta(B)$ has no polar part. Let us then write $\alpha_{k-3}(B)=a_{-1} B^{-1}+a_{0}+a_{1} B+\mathcal{O}\left(B^{2}\right)$, so that $\alpha_{k-3}^{\prime}(B)=-a_{-1} B^{-2}+a_{1}+\mathcal{O}(B)$; similarly, $\beta(B)=b_{0}+b_{1} B+\mathcal{O}\left(B^{2}\right)$ and $\beta^{\prime}(B)=b_{1}+\mathcal{O}(B)$. When computing the finite part of (3.30) we are allowed to commute the finite part operation with the evaluation of $\beta(B=0)=b_{0}$ and $\beta^{\prime}(B=0)=b_{1}$ since the retarded integral of a source term $\propto r^{-2}$ is convergent. The solution $\chi_{k L}$ may then be put in the form

$$
\begin{aligned}
\underset{k}{\chi_{L}} & =\frac{1}{r}\left[\frac{a_{-1}}{2} \ln ^{2}\left(\frac{r}{r_{0}}\right)+a_{0} \ln \left(\frac{r}{r_{0}}\right)+a_{1}\right] \hat{n}_{L} F^{(k-3)}(t-r) \\
& +b_{1} \underset{B=0}{\mathrm{FP}} \square_{R}^{-1}\left[\hat{n}_{L}\left(\frac{r}{r_{0}}\right)^{B} r^{-2} F^{(k-2)}(t-r)\right] \\
& +b_{0} \underset{B=0}{\mathrm{FP}} \square_{R}^{-1}\left[\hat{n}_{L}\left(\frac{r}{r_{0}}\right)^{B} \ln \left(\frac{r}{r_{0}}\right) r^{-2} F^{(k-2)}(t-r)\right]+\mathcal{O}\left(\frac{1}{r^{2-\epsilon}}\right),
\end{aligned}
$$

where

$$
\begin{aligned}
a_{-1} & =\frac{(-)^{k+\ell} 2^{k-3}(k-3) !}{(k-3-\ell) !(k-2+\ell) !}, \\
a_{0} & =a_{-1}\left[H_{k-3-\ell}-H_{k-3}-2 H_{\ell}+H_{k-2+\ell}\right], \\
a_{1} & =\frac{a_{0}^{2}}{2 a_{-1}}+\frac{a_{-1}}{2}\left[H_{k-3-\ell, 2}-H_{k-3,2}+H_{k-2+\ell, 2}\right],
\end{aligned}
$$

together with $b_{0}=2 a_{-1}$ and $b_{1}=2 a_{0}$; here $H_{p, 2}=\sum_{j=1}^{p} \frac{1}{j^{2}}$ denotes the second harmonic number. The formula (3.37) is also sufficient for our purposes, as the asymptotic form of the last two terms can be computed with the help of Eqs. (3.30)-(3.31).

\section{QUARTIC METRIC AND RADIATIVE QUADRUPOLE MOMENT}

Based on the formulas developed in Sec. III A, we have implemented the MPM algorithm, as summarized by Eqs. (2.13)-(2.15), to compute the tails-of-tails cubic metric $h_{M^{2} \times M_{i j}}$ for 
any $r$ greater than the radius of the source. In particular, we have recovered the $1 / r$ asymptotic behaviour (2.16), when $r \rightarrow+\infty$ with $t-r=$ const, obtained in [8]. With $h_{M^{2} \times M_{i j}}$ in hands, using Eqs. (2.17)-(2.18), we computed the quartic source term $\Lambda_{M^{3} \times M_{i j}}$, checked that its divergence is identically zero, and integrated it at leading order when $r \rightarrow$ $+\infty$. Within this stage, we have extensively employed the formulas developed in Secs. III BIII C. ${ }^{8}$

Notice that once we have the quartic source $\Lambda_{M^{3} \times M_{i j}}$, we could use the material from the previous section to determine the comprehensive quartic metric $h_{M^{3} \times M_{i j}}$, but that would be a very long process, since the function $V_{m}$ can be quite complex. Now, it is not simply given by a Legendre function $Q_{m}$, as was the case with the cubic metric $h_{M^{2} \times M_{i j}}$, but is typically a product of combinations of Legendre functions with polynomials and algebraic rational fractions (such that $V_{m} \in \mathscr{V}_{m}$, of course). As we are interested in the radiative quadrupole moment detected at "future null infinity", we content ourselves with the $1 / r$ part of the quartic metric. This allows us to resort to the far-zone version of the MPM algorithm defined in the Appendix B of [8].

Finally, our complete result for the leading $1 / r$ term (actually made of $1 / r, \ln r / r$ and $\ln ^{2} r / r$ terms) of the quartic metric in harmonic coordinates reads

$$
\begin{aligned}
h_{M^{3} \times M_{i j}}^{00}= & \frac{M^{3} \hat{n}_{a b}}{r} \int_{0}^{+\infty} \mathrm{d} \tau M_{a b}^{(6)}\left\{-\frac{8}{3} \ln ^{3}\left(\frac{\tau}{2 r}\right)+\frac{148}{21} \ln ^{2}\left(\frac{\tau}{2 r}\right)+\frac{232}{21} \ln \left(\frac{r}{r_{0}}\right) \ln \left(\frac{\tau}{2 r}\right)\right. \\
& \left.+\frac{1016}{2205} \ln \left(\frac{\tau}{2 r}\right)+\frac{104}{15} \ln \left(\frac{r}{r_{0}}\right)+\frac{16489}{1575}-\frac{232 \pi^{2}}{63}\right\}+\mathcal{O}\left(\frac{1}{r^{2-\epsilon}}\right), \\
h_{M^{3} \times M_{i j}}^{0 i}= & \frac{M^{3} \hat{n}_{a b i}}{r} \int_{0}^{+\infty} \mathrm{d} \tau M_{a b}^{(6)}\left\{-\frac{26}{35} \ln ^{2}\left(\frac{\tau}{2 r}\right)-\frac{8}{105} \ln \left(\frac{\tau}{2 r}\right) \ln \left(\frac{r}{r_{0}}\right)\right. \\
& \left.\quad-\frac{6658}{11025} \ln \left(\frac{\tau}{2 r}\right)+\frac{178}{315} \ln \left(\frac{r}{r_{0}}\right)-\frac{59287}{33075}+\frac{8 \pi^{2}}{315}\right\} \\
+ & \frac{M^{3} \hat{n}_{a}}{r} \int_{0}^{+\infty} \mathrm{d} \tau M_{a i}^{(6)}\left\{-\frac{8}{3} \ln ^{3}\left(\frac{\tau}{2 r}\right)+\frac{562}{75} \ln ^{2}\left(\frac{\tau}{2 r}\right)+\frac{832}{75} \ln \left(\frac{\tau}{2 r}\right) \ln \left(\frac{r}{r_{0}}\right)\right. \\
h_{M^{3} \times M_{i j}}^{i j}= & \frac{M^{3} \hat{n}_{a b i j}}{r} \int_{0}^{+\infty} \mathrm{d} \tau M_{i j}^{(6)}\left\{-\ln ^{2}\left(\frac{\tau}{2 r}\right)-\frac{4}{5} \ln \left(\frac{\tau}{2 r}\right)+\frac{107}{105} \ln \left(\frac{r}{r_{0}}\right)-\frac{30868}{11025}\right\} \\
+ & 2 \frac{M^{3} \hat{n}_{a(j}}{r} \int_{0}^{+\infty} \mathrm{d} \tau M_{i) a}^{(6)}\left\{\frac{234}{35} \ln ^{2}\left(\frac{\tau}{2 r}\right)+\frac{104}{35} \ln \left(\frac{\tau}{2 r}\right) \ln \left(\frac{r}{r_{0}}\right)\right. \\
& \left.+\frac{58694}{3675} \ln \left(\frac{\tau}{2 r}\right)-\frac{598}{735} \ln \left(\frac{r}{r_{0}}\right)+\frac{1487812}{77175}-\frac{104 \pi^{2}}{105}\right\} \\
+ & \frac{M^{3} \hat{n}_{a b} \delta_{i j}}{r} \int_{0}^{+\infty} \mathrm{d} \tau M_{a b}^{(6)}\left\{-\frac{48}{7} \ln ^{2}\left(\frac{\tau}{2 r}\right)-\frac{64}{21} \ln \left(\frac{\tau}{2 r}\right) \ln \left(\frac{r}{r_{0}}\right)\right. \\
& \left.\quad-\frac{7108}{441} \ln \left(\frac{\tau}{2 r}\right)+\frac{1756}{2205} \ln \left(\frac{r}{r_{0}}\right)-\frac{4508029}{231525}+\frac{64 \pi^{2}}{63}\right\}
\end{aligned}
$$

\footnotetext{
${ }^{8}$ For all these calculations we make intensive use of the algebraic computing software Mathematica with the tensor package $x A c t$ [57].
} 


$$
\begin{gathered}
+\frac{M^{3}}{r} \int_{0}^{+\infty} \mathrm{d} \tau M_{i j}^{(6)}\left\{-\frac{8}{3} \ln ^{3}\left(\frac{\tau}{2 r}\right)+\frac{16}{3} \ln ^{2}\left(\frac{\tau}{2 r}\right)+\frac{152}{15} \ln \left(\frac{\tau}{2 r}\right) \ln \left(\frac{r}{r_{0}}\right)\right. \\
\left.-\frac{2332}{525} \ln \left(\frac{\tau}{2 r}\right)+\frac{3608}{525} \ln \left(\frac{r}{r_{0}}\right)+\frac{286408}{55125}-\frac{152 \pi^{2}}{45}\right\}+\mathcal{O}\left(\frac{1}{r^{2-\epsilon}}\right) .
\end{gathered}
$$

The quadrupole moments inside the integrals are evaluated at time $t-r-\tau$. Note that, at this stage, the logarithms involve both the radial distance $r$ to the source and the constant $r_{0}$ coming from the MPM algorithm. We shall now extract the relevant physical information from the above metric as viewed at future null infinity, in the form of the so-called radiative quadrupole moment $U_{i j}[35]$ — not to be confused of course with the source type quadrupole moment $M_{i j}$.

So far, we have performed all our computations in harmonic coordinates $x^{\mu}$. However, this choice of coordinates has the well-known disadvantage that the coordinate cones $t-r$ (where $r=\left|x^{i}\right|$ ) deviate by powers of the logarithm of $r$ from the true space-time characteristics or light cones. As a result, the $1 / r$ expansion of the metric (as $r \rightarrow+\infty$ with $t-r=$ const) involves powers of logarithms. We get rid of them by going to radiative coordinates $X^{\mu}$ for which the associated coordinate cones $T-R$ (where $R=\left|X^{i}\right|$ ) become asymptotically tangent to the true light cones at future null infinity. As in previous works [8], this is achieved by applying the coordinate transformation $X^{\mu}=x^{\mu}+\xi^{\mu}(x)$, where $\xi^{\mu}$ is defined by

$$
\begin{aligned}
\xi^{0} & =-2 M \ln \left(\frac{r}{b_{0}}\right), \\
\xi^{i} & =0,
\end{aligned}
$$

with $b_{0}$ denoting an arbitrary scale that is a priori different from the scale $r_{0}$. Let us show that this simple coordinate change is sufficient to remove all the log-terms from our quartic metric (4.1), so that, in radiative coordinates $X^{\mu}$, it is straightforward to define the radiative quadrupole moment $U_{i j}$. Keeping only the $1 / R$ terms and consistently taking into account all the $M^{3} \times M_{i j}$ interactions, one can check that the metric in radiative coordinates $H_{M^{3} \times M_{i j}}$ differs from the metric $h_{M^{3} \times M_{i j}}$ in harmonic-coordinates by

$$
H_{M^{3} \times M_{i j}}^{\mu \nu}=h_{M^{3} \times M_{i j}}^{\mu \nu}-\xi^{\lambda} \partial_{\lambda} h_{M^{2} \times M_{i j}}^{\mu \nu}+\frac{1}{2} \xi^{\lambda} \xi^{\sigma} \partial_{\lambda \sigma}^{2} h_{M \times M_{i j}}^{\mu \nu}-\frac{1}{6} \xi^{\lambda} \xi^{\sigma} \xi^{\rho} \partial_{\lambda \sigma \rho}^{3} h_{M_{i j}}^{\mu \nu}+\mathcal{O}\left(\frac{1}{R^{2}}\right)
$$

where both sides are evaluated at the same dummy coordinate point, say $X^{\mu}$. Injecting in this relation the results found for $h_{M^{3} \times M_{i j}}, h_{M^{2} \times M_{i j}}, h_{M \times M_{i j}}$ and $h_{M_{i j}}$, recalled in Sec. II, with $\xi^{\mu}$ given by (4.2), we indeed observe that all the logarithms of $R$ vanish. More precisely, we obtain

$$
\begin{aligned}
H_{M^{3} \times M_{i j}}^{00}= & \frac{M^{3} \hat{N}_{a b}}{R} \int_{0}^{+\infty} \mathrm{d} \tau M_{a b}^{(6)}\left\{-\frac{8}{3} \ln ^{3}\left(\frac{\tau}{2 b_{0}}\right)-4 \ln ^{2}\left(\frac{\tau}{2 b_{0}}\right)+\frac{232}{21} \ln \left(\frac{\tau}{2 b_{0}}\right) \ln \left(\frac{\tau}{2 r_{0}}\right)\right. \\
& \left.-\frac{14272}{2205} \ln \left(\frac{\tau}{2 b_{0}}\right)+\frac{104}{15} \ln \left(\frac{\tau}{2 r_{0}}\right)+\frac{16489}{1575}-\frac{232 \pi^{2}}{63}\right\}+\mathcal{O}\left(\frac{1}{R^{2}}\right), \\
H_{M^{3} \times M_{i j}}^{0 i}= & \frac{M^{3} \hat{N}_{a b i}}{R} \int_{0}^{+\infty} \mathrm{d} \tau M_{a b}^{(6)}\left\{-\frac{2}{3} \ln ^{2}\left(\frac{\tau}{2 b_{0}}\right)-\frac{8}{105} \ln \left(\frac{\tau}{2 b_{0}}\right) \ln \left(\frac{\tau}{2 r_{0}}\right)\right.
\end{aligned}
$$




$$
\begin{aligned}
& \left.-\frac{1432}{1225} \ln \left(\frac{\tau}{2 b_{0}}\right)+\frac{178}{315} \ln \left(\frac{\tau}{2 r_{0}}\right)-\frac{59287}{33075}+\frac{8 \pi^{2}}{315}\right\} \\
+ & \frac{M^{3} \hat{N}_{a}}{R} \int_{0}^{+\infty} \mathrm{d} \tau M_{a i}^{(6)}\left\{-\frac{8}{3} \ln ^{3}\left(\frac{\tau}{2 b_{0}}\right)-\frac{18}{5} \ln ^{2}\left(\frac{\tau}{2 b_{0}}\right)+\frac{832}{75} \ln \left(\frac{\tau}{2 b_{0}}\right) \ln \left(\frac{\tau}{2 r_{0}}\right)\right. \\
& \left.-\frac{45448}{7875} \ln \left(\frac{\tau}{2 b_{0}}\right)+\frac{1154}{175} \ln \left(\frac{\tau}{2 r_{0}}\right)+\frac{212134}{18375}-\frac{832 \pi^{2}}{225}\right\}+\mathcal{O}\left(\frac{1}{R^{2}}\right) \\
H_{M^{3} \times M_{i j}}^{i j}= & \frac{M^{3} \hat{N}_{a b i j}}{R} \int_{0}^{+\infty} \mathrm{d} \tau M_{a b}^{(6)}\left\{-\ln ^{2}\left(\frac{\tau}{2 b_{0}}\right)-\frac{191}{105} \ln \left(\frac{\tau}{2 b_{0}}\right)+\frac{107}{105} \ln \left(\frac{\tau}{2 r_{0}}\right)-\frac{30868}{11025}\right\} \\
+ & \frac{M^{3} \hat{N}_{a(j}}{R} \int_{0}^{+\infty} \mathrm{d} \tau M_{i) a}^{(6)}\left\{\frac{26}{7} \ln \left(\frac{\tau}{2 b_{0}}\right)+\frac{104}{35} \ln \left(\frac{\tau}{2 b_{0}}\right) \ln \left(\frac{\tau}{2 r_{0}}\right)\right. \\
+ & \left.\frac{8812}{525} \ln \left(\frac{\tau}{2 b_{0}}\right)-\frac{598}{735} \ln \left(\frac{\tau}{2 r_{0}}\right)+\frac{1487812}{77175}-\frac{104 \pi^{2}}{105}\right\} \\
+ & \frac{M^{3} \hat{N}_{a b} \delta_{i j}}{R} \int_{0}^{+\infty} \mathrm{d} \tau M_{a b}^{(6)}\left\{-\frac{80}{21} \ln ^{2}\left(\frac{\tau}{2 b_{0}}\right)-\frac{64}{21} \ln \left(\frac{\tau}{2 b_{0}}\right) \ln \left(\frac{\tau}{2 r_{0}}\right)\right. \\
& \left.-\frac{592}{35} \ln \left(\frac{\tau}{2 b_{0}}\right)+\frac{1756}{2205} \ln \left(\frac{\tau}{2 r_{0}}\right)-\frac{4508029}{231525}+\frac{64 \pi^{2}}{63}\right\} \\
+ & \frac{M^{3}}{R} \int_{0}^{+\infty} \mathrm{d} \tau M_{i j}^{(6)}\left\{-\frac{8}{3} \ln \left(\frac{\tau}{2 b_{0}}\right)-\frac{24}{5} \ln { }^{2}\left(\frac{\tau}{2 b_{0}}\right)+\frac{152}{15} \ln \left(\frac{\tau}{2 b_{0}}\right) \ln \left(\frac{\tau}{2 r_{0}}\right)\right. \\
& \left.-\frac{396}{35} \ln \left(\frac{\tau}{2 b_{0}}\right)+\frac{3608}{525} \ln \left(\frac{\tau}{2 r_{0}}\right)+\frac{286408}{55125}-\frac{152 \pi^{2}}{45}\right\}+\mathcal{O}\left(\frac{1}{R^{2}}\right), \quad(4.4 \mathrm{c})
\end{aligned}
$$

where the quadrupole moments are evaluated at time $T_{R}-\tau$ in the past, with $T_{R}=T-R$ denoting the retarded time in radiative coordinates.

By definition, the radiative mass and current multipole moments $U_{L}\left(T_{R}\right)$ and $V_{L}\left(T_{R}\right)$ are then the multipolar coefficients that parameterize the transverse-tracefree (TT) projection of the spatial metric in radiative coordinates, at retarded radiative time $T_{R}$, i.e.,

$$
H_{i j}^{\mathrm{TT}}=-\frac{4}{R} \mathcal{P}_{i j k l} \sum_{\ell=2}^{+\infty} \frac{1}{\ell !}\left\{N_{L-2} U_{k l L-2}\left(T_{R}\right)-\frac{2 \ell}{\ell+1} N_{a L-2} \varepsilon_{a b(k} V_{l) b L-2}\left(T_{R}\right)\right\}+\mathcal{O}\left(\frac{1}{R^{2}}\right)
$$

The TT projection operator is given by $\mathcal{P}_{i j k l}=\mathcal{P}_{i k} \mathcal{P}_{j l}-\frac{1}{2} \mathcal{P}_{i j} \mathcal{P}_{k l}$ where $\mathcal{P}_{i j}=\delta_{i j}-N_{i} N_{j}$ represents the projector onto the plane transverse to the unit direction $N_{i}=X_{i} / R$ from the source to the observer. The associated total energy flux $\mathcal{F}=\left(\mathrm{d} E / \mathrm{d} T_{R}\right)^{\mathrm{GW}}$ reads [35]

$$
\mathcal{F}=\sum_{\ell=2}^{+\infty}\left[\frac{(\ell+1)(\ell+2)}{(\ell-1) \ell \ell !(2 \ell+1) ! !}\left(U_{L}^{(1)}\right)^{2}+\frac{4 \ell(\ell+2)}{(\ell-1)(\ell+1) !(2 \ell+1) ! !}\left(V_{L}^{(1)}\right)^{2}\right] .
$$

For the contribution of the tails-of-tails-of-tails part of the radiative metric (4.4) to the radiative quadrupole moment $U_{i j}$, we get

$$
\delta U_{i j}\left(T_{R}\right)=M^{3} \int_{0}^{+\infty} \mathrm{d} \tau M_{i j}^{(6)}\left(T_{R}-\tau\right)\left[\frac{4}{3} \ln ^{3}\left(\frac{\tau}{2 b_{0}}\right)+\frac{11}{3} \ln ^{2}\left(\frac{\tau}{2 b_{0}}\right)+\frac{124627}{11025} \ln \left(\frac{\tau}{2 b_{0}}\right)\right.
$$




$$
\left.-\frac{428}{105} \ln \left(\frac{\tau}{2 b_{0}}\right) \ln \left(\frac{\tau}{2 r_{0}}\right)-\frac{1177}{315} \ln \left(\frac{\tau}{2 r_{0}}\right)+\frac{129268}{33075}+\frac{428}{315} \pi^{2}\right] .
$$

Adding the known quadratic tails and cubic tails-of-tails [8], we obtain the radiative mass quadrupole moment, complete with respect to such tail interactions up to the quartic level:

$$
\begin{array}{r}
U_{i j}\left(T_{R}\right)=M_{i j}^{(2)}\left(T_{R}\right)+\frac{G M}{c^{3}} \int_{0}^{+\infty} \mathrm{d} \tau M_{i j}^{(4)}\left(T_{R}-\tau\right)\left[2 \ln \left(\frac{c \tau}{2 b_{0}}\right)+\frac{11}{6}\right] \\
+\frac{G^{2} M^{2}}{c^{6}} \int_{0}^{+\infty} \mathrm{d} \tau M_{i j}^{(5)}\left(T_{R}-\tau\right)\left[2 \ln ^{2}\left(\frac{c \tau}{2 b_{0}}\right)+\frac{11}{3} \ln \left(\frac{c \tau}{2 b_{0}}\right)\right. \\
\left.\quad-\frac{214}{105} \ln \left(\frac{c \tau}{2 r_{0}}\right)+\frac{124627}{22050}\right] \\
+\frac{G^{3} M^{3}}{c^{9}} \int_{0}^{+\infty} \mathrm{d} \tau M_{i j}^{(6)}\left(T_{R}-\tau\right)\left[\frac{4}{3} \ln ^{3}\left(\frac{c \tau}{2 b_{0}}\right)+\frac{11}{3} \ln ^{2}\left(\frac{c \tau}{2 b_{0}}\right)\right. \\
+\frac{124627}{11025} \ln \left(\frac{c \tau}{2 b_{0}}\right)-\frac{428}{105} \ln \left(\frac{c \tau}{2 b_{0}}\right) \ln \left(\frac{c \tau}{2 r_{0}}\right) \\
\left.-\frac{1177}{315} \ln \left(\frac{c \tau}{2 r_{0}}\right)+\frac{129268}{33075}+\frac{428}{315} \pi^{2}\right]+\mathcal{O}\left(\frac{1}{c^{12}}\right) .
\end{array}
$$

We have restored the powers of $G$ and $c$ to show that the tails-of-tails-of-tails represent a 4.5PN effect in the waveform. They correspond to the most difficult interaction between multipole moments to be computed up to the 4.5PN level. However, there are several other types of interactions that are easier to control and have not been included here. These are for example the non-linear memory integrals [37, 39, 50-55] starting at the 2.5PN order, but also many instantaneous terms, notably at the 4PN order. All these contributions will be systematically investigated in future work.

We have checked that the coefficient of the maximal power of the logarithm in the quartic tails of (4.7)-(4.8) (namely, the cubic logarithm with coefficient 4/3) agrees with the expectation for the dominant iterated infra-red type logarithms, usually factorized out in tail-induced resummed waveforms [15, 58] (see e.g., Sec. (3.1) in [15]).

For future reference let us also recall the radiative mass octupole and current quadrupole radiative moments up to the cubic tails [15]

$$
\begin{gathered}
U_{i j k}\left(T_{R}\right)=M_{i j k}^{(3)}\left(T_{R}\right)+\frac{G M}{c^{3}} \int_{0}^{+\infty} \mathrm{d} \tau M_{i j k}^{(5)}\left(T_{R}-\tau\right)\left[2 \ln \left(\frac{c \tau}{2 b_{0}}\right)+\frac{97}{30}\right] \\
+\frac{G^{2} M^{2}}{c^{6}} \int_{0}^{+\infty} \mathrm{d} \tau M_{i j k}^{(6)}\left(T_{R}-\tau\right)\left[2 \ln ^{2}\left(\frac{c \tau}{2 b_{0}}\right)+\frac{97}{15} \ln \left(\frac{c \tau}{2 b_{0}}\right)\right. \\
\left.-\frac{26}{21} \ln \left(\frac{c \tau}{2 r_{0}}\right)+\frac{13283}{4410}\right]+\mathcal{O}\left(\frac{1}{c^{9}}\right) \\
V_{i j}\left(T_{R}\right)=S_{i j}^{(2)}\left(T_{R}\right)+\frac{G M}{c^{3}} \int_{0}^{+\infty} \mathrm{d} \tau S_{i j}^{(4)}\left(T_{R}-\tau\right)\left[2 \ln \left(\frac{c \tau}{2 b_{0}}\right)+\frac{7}{3}\right] \\
+\frac{G^{2} M^{2}}{c^{6}} \int_{0}^{+\infty} \mathrm{d} \tau S_{i j}^{(5)}\left(T_{R}-\tau\right)\left[2 \ln ^{2}\left(\frac{c \tau}{2 b_{0}}\right)+\frac{14}{3} \ln \left(\frac{c \tau}{2 b_{0}}\right)\right. \\
\left.-\frac{214}{105} \ln \left(\frac{c \tau}{2 r_{0}}\right)-\frac{26254}{11025}\right]+\mathcal{O}\left(\frac{1}{c^{9}}\right) .
\end{gathered}
$$




\section{ENERGY FLUX OF COMPACT BINARIES ON CIRCULAR ORBITS}

In this section we derive, based on the quartic radiative mass quadrupole moment (4.8), the complete 4.5PN coefficient of the gravitational-wave energy flux (4.6), in the case of binary systems of non-spinning compact objects moving on circular orbits. We thus extend the circular energy flux known at the $3.5 \mathrm{PN}$ order [7-10] by including the 4.5PN coefficient, while the determination of the $4 \mathrm{PN}$ coefficient is left to future work. The test mass limit of our new $4.5 \mathrm{PN}$ coefficient turns out to be in perfect agreement with the prediction from black-hole perturbation theory [44, 48, 49].

The reason why we are able to control the 4.5PN order without knowing the complete $4 \mathrm{PN}$ field (since the source moments are known only up to the 3.5PN order [13-15]) is the fact that for half-integral PN orders, i.e., $\frac{n}{2} \mathrm{PN}$ orders where $n$ is an odd integer, any instantaneous or "non-hereditary" term is zero in the energy flux for circular orbits. This can be shown by a simple dimensional argument (see the discussion in Sec. II of Ref. [59]). Notice that memory effects do not arise at half-integral PN orders, since the time derivative acting on the radiative moments in the flux equation (4.6) turns them into instantaneous quantities. Hence, at the 4.5PN order, only truly "hereditary" tail integrals do contribute to the circular energy flux. It is therefore sufficient to control the occurrence of such hereditary integrals, i.e., of non-linear tail interactions between multipole moments. For that purpose, it is very useful to apply some "selection rules" that permit one to determine all the possible multipole interactions occurring at a given PN order [13-15] (see in particular Sec. III of [15]).

According to those selection rules, in order to control the 4.5PN order for circular orbits, we need only the contributions of (i) quadratic multipole tails, of the form $M \times M_{L}$ or $M \times S_{L}$, with $2 \leqslant \ell \leqslant 5$ for mass moments $M_{L}$ and $2 \leqslant \ell \leqslant 4$ for current moments $S_{L}$, (ii) the quartic quadrupole tails-of-tails-of-tails $M^{3} \times M_{i j}$ (with $\ell=2$ in this case), and (iii) the double product between the quadratic quadrupole tails $M \times M_{i j}$ and the cubic quadrupole tails-of-tails $M^{2} \times M_{i j}$.

The cubic tails-of-tails $M^{2} \times M_{i j}$ by themselves contribute to the circular energy flux, starting at the 3PN order [8]. The 4PN order correction will be given in Eq. (5.7). Moreover, from the point (iii) above, we see that the cubic tails-of-tails also contribute at the $4.5 \mathrm{PN}$ order through their interactions with the quadrupole tails $M \times M_{i j}$.

The computation of quadratic tails for circular compact binaries is classic and will not be detailed. Suffice it to say that, at the 4.5PN order, we need the mass quadrupole moment at $3 \mathrm{PN}$ order, since $4.5 \mathrm{PN}$ means $3 \mathrm{PN}$ beyond the dominant quadrupole tail at the $1.5 \mathrm{PN}$ order. The 3PN quadrupole moment for circular orbits reads (see e.g., Ref. [13]) ${ }^{9}$

$$
M_{i j}=m \nu\left(A x^{\langle i j\rangle}+B \frac{r^{2}}{c^{2}} v^{\langle i j\rangle}+\frac{48}{7} \frac{G^{2} m^{2} \nu}{c^{5} r} x^{\langle i} v^{j\rangle}\right)+\mathcal{O}\left(\frac{1}{c^{7}}\right) .
$$

\footnotetext{
${ }^{9}$ Here $x^{i}$ and $v^{i}$ denote the orbital separation and relative velocity of the two particles (and the angular brackets refer to the STF projection). The mass parameters are the total mass $m=m_{1}+m_{2}$ and the symmetric mass ratio $\nu=\frac{m_{1} m_{2}}{\left(m_{1}+m_{2}\right)^{2}}$. The harmonic-coordinates PN parameter is $\gamma=\frac{G m}{r c^{2}}$, where $r=\left|x^{i}\right|$ represents the radial harmonic-coordinates separation. The quasi-invariant PN parameter is $x=\left(\frac{G m \omega}{c^{3}}\right)^{2 / 3}$ where $\omega$ stands for the orbital frequency, related to $r$ by Eqs. (5.3). A scalar such as the circular energy flux is quasi-invariant when expressed in terms of $x$, in the sense that it stays invariant under the class of coordinate transformations that are asymptotically Minkowskian at infinity.
} 
In order to control the tails at the 4.5PN order we may ignore the above 2.5PN dissipative term since it contributes only at the 4PN order. Notice that the "canonical" quadrupole moment $M_{i j}$ agrees for circular orbits, up to the 3PN order, with the alternative definition of the "source" quadrupole moment $I_{i j}$ [13]. The two coefficients $A$ and $B$ are given by the following expansion series in the PN parameter $\gamma$ :

$$
\begin{aligned}
A & =1+\gamma\left(-\frac{1}{42}-\frac{13}{14} \nu\right)+\gamma^{2}\left(-\frac{461}{1512}-\frac{18395}{1512} \nu-\frac{241}{1512} \nu^{2}\right) \\
& +\gamma^{3}\left(\frac{395899}{13200}-\frac{428}{105} \ln \left(\frac{r}{r_{0}}\right)+\left[\frac{3304319}{166320}-\frac{44}{3} \ln \left(\frac{r}{r_{0}^{\prime}}\right)\right] \nu+\frac{162539}{16632} \nu^{2}+\frac{2351}{33264} \nu^{3}\right) \\
B & =\frac{11}{21}-\frac{11}{7} \nu+\gamma\left(\frac{1607}{378}-\frac{1681}{378} \nu+\frac{229}{378} \nu^{2}\right) \\
& +\gamma^{2}\left(-\frac{357761}{19800}+\frac{428}{105} \ln \left(\frac{r}{r_{0}}\right)-\frac{92339}{5544} \nu+\frac{35759}{924} \nu^{2}+\frac{457}{5544} \nu^{3}\right)
\end{aligned}
$$

Notice the two scales entering the logarithmic terms at the 3PN order: one is the length scale $r_{0}$ coming from the MPM algorithm (see Sec. II), while the other scale $r_{0}^{\prime}$ is the logarithmic barycenter of two gauge constants $r_{1}^{\prime}$ and $r_{2}^{\prime}$ which appear in the 3PN equations of motion in harmonic coordinates [26], i.e., $m \ln r_{0}^{\prime}=m_{1} \ln r_{1}^{\prime}+m_{2} \ln r_{2}^{\prime}$. The latter constant $r_{0}^{\prime}$ thus parameterizes the relation between the orbital frequency $\omega$ for circular orbits and the separation $r$ at the 3PN order in harmonic coordinates, namely

$$
\begin{aligned}
\omega^{2}=\frac{G m}{r^{3}} & \left\{1+(-3+\nu) \gamma+\left(6+\frac{41}{4} \nu+\nu^{2}\right) \gamma^{2}\right. \\
+ & \left.\left(-10+\left[-\frac{75707}{840}+\frac{41}{64} \pi^{2}+22 \ln \left(\frac{r}{r_{0}^{\prime}}\right)\right] \nu+\frac{19}{2} \nu^{2}+\nu^{3}\right) \gamma^{3}+\mathcal{O}\left(\frac{1}{c^{8}}\right)\right\} \\
\gamma=x & \left\{1+\left(1-\frac{\nu}{3}\right) x+\left(1-\frac{65}{12} \nu\right) x^{2}\right. \\
+ & \left.\left(1+\left[-\frac{2203}{2520}-\frac{41}{192} \pi^{2}-\frac{22}{3} \ln \left(\frac{r}{r_{0}^{\prime}}\right)\right] \nu+\frac{229}{36} \nu^{2}+\frac{1}{81} \nu^{3}\right) x^{3}+\mathcal{O}\left(\frac{1}{c^{8}}\right)\right\} .
\end{aligned}
$$

Here, we do not consider the $2.5 \mathrm{PN}$ radiation reaction term in the equations of motion, since it generates some contribution at the $4 \mathrm{PN}$ order but not at the $4.5 \mathrm{PN}$ order. To summarize, there are three arbitrary length scales in the problem: $r_{0}, r_{0}^{\prime}$, as well as $b_{0}$ which originates from our choice of radiative type coordinate system through Eqs. (4.2). It is non trivial to check that these three scales cancel out in the final gauge invariant expression of the energy flux for circular orbits.

Another important step of the calculation is the reduction of the tail integrals to circular orbits. As usual, those integrals are to be computed proceeding as if the worldlines in the integrands obeyed the current circular dynamics, which amounts to neglecting the evolution in the past by radiation reaction. The influence of the past evolution would be to correct the dominant $1.5 \mathrm{PN}$ tail integral by a $2.5 \mathrm{PN}$ radiation reaction term, and would thus be of order $4 \mathrm{PN}$, but not 4.5PN. From Eq. (4.8), we see that, at the $4.5 \mathrm{PN}$ order, we also need some integration formulas involving up to three powers of logarithms. Those are [56]

$$
\int_{0}^{+\infty} \mathrm{d} \tau \ln \left(\frac{\tau}{\tau_{0}}\right) e^{-\mathrm{i} \Omega \tau}=\frac{\mathrm{i}}{\Omega}\left(\ln \left(\Omega \tau_{0}\right)+\gamma_{\mathrm{E}}+\mathrm{i} \frac{\pi}{2}\right),
$$




$$
\begin{aligned}
& \int_{0}^{+\infty} \mathrm{d} \tau \ln ^{2}\left(\frac{\tau}{\tau_{0}}\right) e^{-\mathrm{i} \Omega \tau}=-\frac{\mathrm{i}}{\Omega}\left[\left(\ln \left(\Omega \tau_{0}\right)+\gamma_{\mathrm{E}}+\mathrm{i} \frac{\pi}{2}\right)^{2}+\frac{\pi^{2}}{6}\right], \\
& \int_{0}^{+\infty} \mathrm{d} \tau \ln ^{3}\left(\frac{\tau}{\tau_{0}}\right) e^{-\mathrm{i} \Omega \tau}=\frac{\mathrm{i}}{\Omega}\left[\left(\ln \left(\Omega \tau_{0}\right)+\gamma_{\mathrm{E}}+\mathrm{i} \frac{\pi}{2}\right)^{3}+\frac{\pi^{2}}{2}\left(\ln \left(\Omega \tau_{0}\right)+\gamma_{\mathrm{E}}+\mathrm{i} \frac{\pi}{2}\right)+2 \zeta(3)\right] .
\end{aligned}
$$

Here, $\Omega$ denotes a multiple of the orbital frequency $\omega$. The constant $\tau_{0}$ is arbitrary, and related either to $r_{0}, r_{0}^{\prime}$ or $b_{0}$. We denote by $\gamma_{\mathrm{E}} \simeq 0.577$ the Euler constant, whereas $\zeta(3) \simeq$ 1.202 is the Apéry constant ( $\zeta$ being the usual notation for the Riemann zeta function).

Let us decompose the tail contributions to the energy flux of circular binaries up to the 4.5PN order - as defined by (4.6) in terms of the radiative moments - into those generated by quadratic, cubic and quartic tails,

$$
\mathcal{F}_{\text {tail }}=\mathcal{F}_{\text {quadratic }}+\mathcal{F}_{\text {cubic }}+\mathcal{F}_{\text {quartic }}+\mathcal{O}\left(G^{5}\right)
$$

where the remainder contains the neglected MPM approximations [see Eq. (2.1)]. The quadratic tails correspond to multipole interactions $M \times M_{L}$ and $M \times S_{L}$ (see e.g., Eqs. (3.6)(3.7) in [15]). In particular, we need the full 3PN precision for the mass quadrupole moment, as in Eqs. (5.1)-(5.2). The higher order moments require some lower PN precision. Their explicit expressions can be found in Ref. [13]. It is also worthy to note that the quadratic tails contribute only to half-integral PN approximations. The result in terms of the PN parameter $\gamma=G m /\left(r c^{2}\right)$ up to the $4.5 \mathrm{PN}$ order reads (factorizing out the Newtonian flux as usual)

$$
\begin{gathered}
\mathcal{F}_{\text {quadratic }}=\frac{32 c^{5}}{5 G} \nu^{2} \gamma^{5}\left\{4 \pi \gamma^{3 / 2}\right. \\
+\left(-\frac{25663}{672}-\frac{125}{8} \nu\right) \pi \gamma^{5 / 2}+\left(\frac{90205}{576}+\frac{505747}{1512} \nu+\frac{12809}{756} \nu^{2}\right) \pi \gamma^{7 / 2} \\
+\left(\frac{9997778801}{106444800}-\frac{6848}{105} \ln \left(\frac{r}{r_{0}}\right)+\left[-\frac{8058312817}{2661120}+\frac{287}{32} \pi^{2}+\frac{572}{3} \ln \left(\frac{r}{r_{0}^{\prime}}\right)\right] \nu\right. \\
\left.\left.\quad-\frac{12433367}{13824} \nu^{2}-\frac{1026257}{266112} \nu^{3}\right) \pi \gamma^{9 / 2}+\mathcal{O}\left(\frac{1}{c^{11}}\right)\right\} .
\end{gathered}
$$

In contrast to the quadratic tails, the cubic tails-of-tails contribute to integral PN approximations, starting at the $3 \mathrm{PN}$ order [8]. At the next $4 \mathrm{PN}$ order they involve the contribution of the mass quadrupole moment (to be computed with 1PN precision), as well as that of the mass octupole and current quadrupole moments given by Eqs. (4.9). Furthermore, at the same level of the cubic tails, we must include in the flux the square of the quadratic tails. Those various contributions have all been computed. For their sum, we obtain, extending Eq. (5.9) of [8],

$$
\begin{gathered}
\mathcal{F}_{\text {cubic }}=\frac{32 c^{5}}{5 G} \nu^{2} \gamma^{5}\left\{\left(-\frac{116761}{3675}+\frac{16}{3} \pi^{2}-\frac{1712}{105} \gamma_{\mathrm{E}}-\frac{856}{105} \ln (16 \gamma)+\frac{1712}{105} \ln \left(\frac{r}{r_{0}}\right)\right) \gamma^{3}\right. \\
+\left(\frac{12484937}{30870}-\frac{4040}{63} \pi^{2}+\frac{86456}{441} \gamma_{\mathrm{E}}+\frac{43228}{441} \ln (16 \gamma)-\frac{86456}{441} \ln \left(\frac{r}{r_{0}}\right)\right. \\
+(1-4 \nu)\left[\frac{670000393}{7408800}+\frac{445}{42} \pi^{2}-\frac{56731}{4410} \gamma_{\mathrm{E}}-\frac{56731}{8820} \ln (16 \gamma)\right.
\end{gathered}
$$




$$
\left.\left.\left.+\frac{133771}{4410} \ln 2-\frac{47385}{1568} \ln 3+\frac{56731}{4410} \ln \left(\frac{r}{r_{0}}\right)\right]\right) \gamma^{4}+\mathcal{O}\left(\frac{1}{c^{10}}\right)\right\} .
$$

The constant $b_{0}$ disappears, as expected. However, $\mathcal{F}_{\text {cubic }}$ still contains $r_{0}$. The point is that these cubic tails at the $3 \mathrm{PN}$ and $4 \mathrm{PN}$ orders are not the only contributions to the full coefficients, since the flux also contains many instantaneous (non-tails) terms that depend on the source multipole moments, and notably the 4PN quadrupole. After the moments have been replaced by their explicit expressions, those terms should cancel out the remaining constants $r_{0}$ in (5.7). Thus, since the $4 \mathrm{PN}$ instantaneous contributions are not known, we shall ignore henceforth the 4PN coefficient in the flux except for the partial result (5.7).

In addition, there are other tail contributions at the $4 \mathrm{PN}$ order (but not at the $4.5 \mathrm{PN}$ order) that we have not yet taken into account. We can mention for instance the coupling between the dominant $1.5 \mathrm{PN}$ tail term and the $2.5 \mathrm{PN}$ non-linear memory effect, which is therefore expected to contribute at the $4 \mathrm{PN}$ order. Moreover, there exists a $2.5 \mathrm{PN}$ effect corresponding to the past evolution of the binary source due to radiation reaction. It should affect the computation of the $1.5 \mathrm{PN}$ tail integral at the $4 \mathrm{PN}$ order. We also recall the nonlocal 4PN tail term entering the equations of motion [20,21], which will have to be included when performing the order reduction of accelerations coming from the time derivatives of the Newtonian quadrupole moment. All these contributions will have to be systematically included in future work.

Let us next focus on the computation of the quartic-order tails in the flux. One contribution is directly due to the quartic tail term at the $4.5 \mathrm{PN}$ order in the radiative mass quadrupole moment (4.8). However, there is another contribution coming from a double product between the quadratic quadrupole tail at the $1.5 \mathrm{PN}$ order and the cubic quadrupole tail-of-tail at the 3PN order - recall that the energy flux contains the square of the time derivative of Eq. (4.8). It turns out that important cancellations occur between these two terms, notably all the logarithms squared and cubed disappear, leaving only a term linear in the logarithm. The constant $b_{0}$ cancels out as expected, but a dependence of $r_{0}$ is left out at this stage. In the end, we find that

$$
\begin{aligned}
\mathcal{F}_{\text {quartic }}=\frac{32 c^{5}}{5 G} \nu^{2} \gamma^{5}\{ & \left(-\frac{467044}{3675}-\frac{3424}{105} \ln (16 \gamma)+\frac{6848}{105} \ln \left(\frac{r}{r_{0}}\right)-\frac{6848}{105} \gamma_{\mathrm{E}}\right) \pi \gamma^{9 / 2} \\
& \left.+\mathcal{O}\left(\frac{1}{c^{11}}\right)\right\} .
\end{aligned}
$$

Finally we are in a position to control the half-integral PN approximations (or so-called "odd" PN terms) in the energy flux for circular orbits, as they are entirely due to tail integrals. The "odd" part of the flux in this case is

$$
\left.\mathcal{F}\right|_{\text {odd }}=\left.\mathcal{F}_{\text {tail }}\right|_{\text {odd }}=\left.\left(\mathcal{F}_{\text {quadratic }}+\mathcal{F}_{\text {quartic }}\right)\right|_{\text {odd }}+\mathcal{O}\left(G^{5}\right)
$$

We do not include the cubic tail part (5.7) since it is "even" in the PN sense. Therefore, we need only to sum up Eqs. (5.6) and (5.8). We gladly discover that the scale $r_{0}$ cancels out from the sum, thereby obtaining

$$
\left.\mathcal{F}\right|_{\text {odd }}=\frac{32 c^{5}}{5 G} \nu^{2} \gamma^{5}\left\{4 \pi \gamma^{3 / 2}\right.
$$




$$
\begin{gathered}
+\left(-\frac{25663}{672}-\frac{125}{8} \nu\right) \pi \gamma^{5 / 2}+\left(\frac{90205}{576}+\frac{505747}{1512} \nu+\frac{12809}{756} \nu^{2}\right) \pi \gamma^{7 / 2} \\
+\left(-\frac{24709653481}{745113600}-\frac{6848}{105} \gamma_{\mathrm{E}}-\frac{3424}{105} \ln (16 \gamma)\right. \\
+\left[-\frac{8058312817}{2661120}+\frac{287}{32} \pi^{2}+\frac{572}{3} \ln \left(\frac{r}{r_{0}^{\prime}}\right)\right] \nu \\
\left.\left.-\frac{12433367}{13824} \nu^{2}-\frac{1026257}{266112} \nu^{3}\right) \pi \gamma^{9 / 2}+\mathcal{O}\left(\frac{1}{c^{11}}\right)\right\}
\end{gathered}
$$

Still there remains a dependence on the scale $r_{0}^{\prime}$ coming from the equations of motion, but that is merely due to our use of the harmonic-coordinates PN parameter $\gamma$. Eliminating $\gamma$ in favor of the quasi-invariant frequency-related PN parameter $x=\left(G m \omega / c^{3}\right)^{2 / 3}$ with the help of Eqs. (5.3) yields then our final result:

$$
\begin{aligned}
\left.\mathcal{F}\right|_{\text {odd }}=\frac{32 c^{5}}{5 G} \nu^{2} x^{5} & \left\{4 \pi x^{3 / 2}\right. \\
& +\left(-\frac{8191}{672}-\frac{583}{24} \nu\right) \pi x^{5 / 2}+\left(-\frac{16285}{504}+\frac{214745}{1728} \nu+\frac{193385}{3024} \nu^{2}\right) \pi x^{7 / 2} \\
& +\left(\frac{265978667519}{745113600}-\frac{6848}{105} \gamma_{\mathrm{E}}-\frac{3424}{105} \ln (16 x)+\left[\frac{2062241}{22176}+\frac{41}{12} \pi^{2}\right] \nu\right. \\
& \left.\left.\quad-\frac{133112905}{290304} \nu^{2}-\frac{3719141}{38016} \nu^{3}\right) \pi x^{9 / 2}+\mathcal{O}\left(\frac{1}{c^{11}}\right)\right\}
\end{aligned}
$$

We insist that the latter odd part of the flux, although it has been computed only from tail contributions, represents the full PN-odd part of the complete flux, in the case of circular orbits. Thus, the coefficients can be compared with those derived from black-hole perturbation theory in the small mass ratio limit $\nu \rightarrow 0$. Black-hole perturbations have been expanded for this problem at the $1.5 \mathrm{PN}$ order [40], then extended up to the 5.5PN order in [41-44], and more recently, using the method [45-47], up to extremely high PN orders [48, 49]. Our new 4.5PN result in Eq. (5.11) perfectly reproduces the latter works in the limit where $\nu \rightarrow 0$ (see Eq. (3.1) in [44]).

\section{CONCLUSION}

This paper is a contribution to our current program to provide 4.5PN accurate gravitational waveforms (together with the orbital phasing) generated by the inspiral of compact binary systems without spins. A first part of this program, concerning the $4 \mathrm{PN}$ accurate equations of motion, has already been completed [20,21]. Here, we solve one of the main difficulties regarding the $4.5 \mathrm{PN}$ wave field, namely the computation of quartic non-linearities associated with high order tail effects called "tails-of-tails-of-tails". These terms correspond to the interaction between three mass monopoles $M$ and the quadrupole moment $M_{i j}$. They contribute at the 4.5PN order to the asymptotic waveform and gravitational energy flux (beyond the Einstein quadrupole formula).

Our calculation is based on the multipolar-post-Minkowskian (MPM) algorithm for solving the Einstein field equations in the exterior region of a general isolated source [8, 38, 39]. We developed new mathematical formulas to express the retarded solutions of d'Alembertian 
equations sourced by certain non-linear tail (or "hereditary") integrals. Such formulas are necessary in practice for implementing the non-linear iterations leading to the quartic metric, and allow us to express our results in essentially analytic closed form. Those formulas involve a machinery of Legendre polynomials and associated Legendre functions.

We have thoroughly computed the cubic-order tails-of-tails at any distance from the source (while only the leading asymptotic terms were previously known [8]), and plugged the latter piece of the gravitational field into the source term in order to perform the next iteration of the metric at the quartic level. From that source term, we derived, resorting again to formulas generalized from previous works, the tails-of-tails-of-tails at the leading order in the inverse distance to the source. Our main result is encapsulated in the radiative mass quadrupole moment (4.8), which describes the quadrupolar gravitational waves at infinity, up to the level of the quartic interaction $M^{3} \times M_{i j}$ which contributes at the $4.5 \mathrm{PN}$ order in the asymptotic waveform.

As an application, we computed the total energy flux emitted by compact binary systems in the case of circular orbits. After extending the classic calculation of quadratic tails up to the 4.5PN order, we obtained the new contributions due to the quartic tails-of-tails-of-tails. We also extended the computation of cubic tails-of-tails at the 4PN order. This led us to the complete 4.5PN coefficient in the energy flux of compact binaries on circular orbits, as given by Eq. (5.11). The energy flux represents an essential theoretical input for gravitationalwave data analysis, since it drives the orbital phase evolution [3, 4]. Notice, however, that the $4 \mathrm{PN}$ coefficient is not yet known, except in the test mass limit. Its computation is left for future work. Finally, the test-particle limit of our 4.5PN expression (when $\nu \rightarrow 0$ ) is in perfect agreement with the result found by means of black-hole perturbation methods applied to the two body problem [44, 48, 49].

\section{Appendix A: Proofs of some mathematical formulas}

\section{Formula (3.11)}

We want to prove that, for any $\ell \geqslant k-2, m \geqslant k-2$, and for any $V_{m} \in \mathscr{V}_{m}$,

$$
\begin{aligned}
\underset{k, m}{\Psi_{L}}=-\hat{n}_{L} \int_{1}^{+\infty} & \mathrm{d} s F^{(k-2)}(t-r s) \\
& \times\left[Q_{\ell}(s) \int_{1}^{s} \mathrm{~d} y V_{m}^{(-k+2)}(y) P_{\ell}(y)+P_{\ell}(s) \int_{s}^{+\infty} \mathrm{d} y V_{m}^{(-k+2)}(y) Q_{\ell}(y)\right],
\end{aligned}
$$

where $V_{m}^{(-k+2)}(y)$ is the $(k-2)$-th anti-derivative of $V_{m}(y)$ defined by Eq. (3.10). We will proceed by induction over the integer $k$. Let us thus assume that (A1) is valid up to $k-1$ with $k \geqslant 3$, and let us show that it is then valid for $k$. By definition, we have

$$
\underset{k, m}{\Psi_{L}}=\underset{B=0}{\mathrm{FP}} \square_{R}^{-1}\left[\hat{n}_{L}\left(\frac{r}{r_{0}}\right)^{B} r^{-k} \int_{1}^{+\infty} \mathrm{d} y V_{m}(y) F(t-r y)\right],
$$

which we can integrate by part, for $V_{m} \in \mathscr{V}_{m}$ and $m \geqslant k-2$. We find

$$
\underset{k, m}{\Psi_{L}}=\underset{B=0}{\mathrm{FP}} \square_{R}^{-1}\left[\hat{n}_{L}\left(\frac{r}{r_{0}}\right)^{B} r^{-k+1} \int_{1}^{+\infty} \mathrm{d} y V_{m}^{(-1)}(y) F^{(1)}(t-r y)\right],
$$


where $V_{m}^{(-1)}(y)=\int_{1}^{y} \mathrm{~d} x V_{m}(x)$ in agreement with (3.10). As $m \geqslant 1$ (because $k \geqslant 3$ ), $\alpha_{m}=$ $\int_{1}^{+\infty} \mathrm{d} y V_{m}(y)$ is a convergent integral. Posing $\tilde{V}_{m-1}(y)=V_{m}^{(-1)}(y)-\alpha_{m}$, we rewrite (A3) as

$$
\begin{aligned}
\underset{k, m}{\Psi_{L}} & =\alpha_{m} \underset{B=0}{\operatorname{FP}} \square_{R}^{-1}\left[\hat{n}_{L}\left(\frac{r}{r_{0}}\right)^{B} r^{-k} F(t-r)\right] \\
& +\underset{B=0}{\mathrm{FP}} \square_{R}^{-1}\left[\hat{n}_{L}\left(\frac{r}{r_{0}}\right)^{B} r^{-k+1} \int_{1}^{+\infty} \mathrm{d} y \tilde{V}_{m-1}(y) F^{(1)}(t-r y)\right] .
\end{aligned}
$$

The point now is that $\tilde{V}_{m-1} \in \mathscr{V}_{m-1}$, so that we can make use of Eq. (A1) (which is our induction hypothesis) to obtain

$$
\begin{aligned}
\underset{k, m}{\Psi_{L}}=\alpha_{m} \underset{B=0}{\mathrm{FP}} \square_{R}^{-1}\left[\hat{n}_{L}\left(\frac{r}{r_{0}}\right)^{B} r^{-k} F(t-r)\right] \\
-\hat{n}_{L} \int_{1}^{+\infty} \mathrm{d} s F^{(k-2)}(t-r s) \\
\times\left[Q_{\ell}(s) \int_{1}^{s} \mathrm{~d} y \tilde{V}_{m-1}^{(-k+3)}(y) P_{\ell}(y)+P_{\ell}(s) \int_{s}^{+\infty} \mathrm{d} y \tilde{V}_{m-1}^{(-k+3)}(y) Q_{\ell}(y)\right] .
\end{aligned}
$$

The first term is instantaneous and, keeping in mind that $\ell \geqslant k-2$, it may be integrated by means of the formula (A.11) of [39]. Since we have $\tilde{V}_{m-1}^{(-k+3)}(y)=V_{m}^{(-k+2)}(y)-\alpha_{m} \frac{(y-1)^{k-3}}{(k-3) !}$, we obtain exactly the result ${ }_{k, m} \Psi_{L}$ given by (A1) that we wanted to prove, with however the following additional term

$$
\begin{aligned}
& \underset{k, m}{\delta \Psi_{L}}=\alpha_{m} \hat{n}_{L} \int_{1}^{+\infty} \mathrm{d} s F^{(k-2)}(t-r s) \\
& \times\left[\mathcal{A}_{\ell}^{k-3}(s)-\frac{2^{k-3}(k-3) !(\ell+2-k) !}{(\ell+k-2) !} \sum_{j=0}^{k-3} \frac{(\ell+j) !(s-1)^{j}}{2^{j}(\ell-j) !(j !)^{2}}\right],
\end{aligned}
$$

in which we have introduced the following combination

$$
\mathcal{A}_{\ell}^{k-3}(s)=Q_{\ell}(s) \int_{1}^{s} \mathrm{~d} y P_{\ell}(y) \frac{(y-1)^{k-3}}{(k-3) !}+P_{\ell}(s) \int_{s}^{+\infty} \mathrm{d} y Q_{\ell}(y) \frac{(y-1)^{k-3}}{(k-3) !} .
$$

We are now going to prove that the additional term (A6) is actually zero, because the quantity in the square brackets of (A6) is in fact identically zero (for any $s \in] 1,+\infty[$ ). To this end, we notice that the two integrals appearing in (A7) are of the same type, namely

$$
\mathcal{I}_{\ell}^{p}(s)=\int_{a}^{s} \mathrm{~d} y f_{\ell}(y) \frac{(y-1)^{p}}{p !}
$$

where we have posed $p=k-3$ for simplicity sake. The lower boundary is $a=1$ or $a=+\infty$ according to the integral in (A7) we are considering. The function $f_{\ell}(y)$ represents either the Legendre polynomial $P_{\ell}(y)$ for $a=1$ or the Legendre function $Q_{\ell}(y)$ for $a=+\infty$. In both cases, the integral is well-defined. Using the fact that $f_{\ell}(y)$ satisfies the usual Legendre 
differential equation ${ }^{10}$ and performing two integrations by parts, we obtain the following recursive relation

$$
\mathcal{I}_{\ell}^{p}(s)=\frac{1}{(\ell-p)(\ell+p+1)}\left[\left(f_{\ell}(s)[(\ell-p) s-p]-\ell f_{\ell-1}(s)\right) \frac{(s-1)^{p}}{p !}+2 p \mathcal{I}_{\ell}^{p-1}(s)\right]
$$

It nicely translates, when inserted into (A7), into the simple recurrence equation

$$
\mathcal{A}_{\ell}^{p}(s)=\frac{1}{(\ell-p)(\ell+p+1)}\left[\frac{(s-1)^{p}}{p !}+2 p \mathcal{A}_{\ell}^{p-1}(s)\right]
$$

whose solution is straightforwardly found to be

$$
\mathcal{A}_{\ell}^{p}(s)=\frac{2^{p} p !(\ell-p-1) !}{(\ell+p+1) !} \sum_{j=0}^{p} \frac{(\ell+j) !(s-1)^{j}}{2^{j}(\ell-j) !(j !)^{2}} .
$$

To arrive at the latter expression, we need the readily checked relation $A_{\ell}^{0}(s)=\frac{1}{\ell(\ell+1)}$, which plays the role of normalization condition. The result (A11) shows that the additional term (A6) is indeed zero, which completes our proof of Eq. (A1).

\section{Formulas (3.12) and (3.13)}

We notice that, for the proof of the case where $\ell=0, k=3$ and $m=2$, all the reasonings in the previous section A 1 remain valid for $\ell=0$ up to Eq. (A5), which is actually true as soon as $m \geqslant 1$. Now, the equation (A.13) of [39] tells us that

$$
\underset{B=0}{\mathrm{FP}} \square_{R}^{-1}\left[\left(\frac{r}{r_{0}}\right)^{B} r^{-3} F(t-r)\right]=-\left[\ln \left(\frac{r}{r_{0}}\right)+1\right] \frac{F(t-r)}{r}+2 \int_{1}^{+\infty} \mathrm{d} s F^{(1)}(t-r s) Q_{0}(s) .
$$

Inserting (A12) into (A5) for $k=3$, we readily recover the result (3.12) in the text.

The generalization to the case where $\ell=0, k \geqslant 3$ and $m \geqslant k-2$, given by the formula (3.13) in the text, differs from the proof we just presented for $k=3$, and goes as follows. First, using the definition (6.3) of Ref. [38], we pose

$$
R_{B}(r, s)=\frac{1}{2} \int_{0}^{r} \mathrm{~d} x\left(\frac{x}{r_{0}}\right)^{B} x^{-k+1} \int_{1}^{+\infty} \mathrm{d} y V_{m}(y) F[s-x(y-1)] .
$$

10 Namely,

$$
\frac{\mathrm{d}}{\mathrm{d} y}\left[\left(1-y^{2}\right) \frac{\mathrm{d} f_{\ell}(y)}{\mathrm{d} y}\right]+\ell(\ell+1) f_{\ell}(y)=0 .
$$

We remind also the following properties of Legendre functions that are used in our computation:

$$
\begin{aligned}
& \left(1-y^{2}\right) \frac{\mathrm{d} f_{\ell}(y)}{\mathrm{d} y}=\ell\left[f_{\ell-1}(y)-y f_{\ell}(y)\right], \\
& P_{\ell}(y) Q_{\ell-1}(y)-P_{\ell-1}(y) Q_{\ell}(y)=\frac{1}{\ell} .
\end{aligned}
$$


Then, by virtue of the theorem (6.1) of [36], we can write

$$
\underset{k, m}{\Psi_{L=0}}=\frac{1}{r} \mathrm{FP}_{B=0} \int_{-\infty}^{t-r} \mathrm{~d} s\left[R_{B}\left(\frac{t-r-s}{2}, s\right)-R_{B}\left(\frac{t+r-s}{2}, s\right)\right] .
$$

Let us call $A_{1}$ the first term in this expression, actually a retarded homogeneous solution of the wave equation, and $A_{2}$ the second term, which is made of a mixture of retarded and advanced times. By construction, we have ${ }_{k, m} \Psi_{L=0}=A_{1}+A_{2}$.

We inject (A13) into $A_{1}$ and define the new variable $u=\frac{t-r-s}{2}$, thereby expressing $A_{1}$ in terms of the new set of variables $(u, x, y)$. Next, after commuting the $x$ and $u$ integrals, we explicitly integrate over $u$. Once this is done, we perform $k-1$ integrations by part with respect to $x$ and apply the finite part procedure to get

$$
A_{1}=\frac{(-)^{k}}{2(k-2) !} \frac{1}{r} \int_{0}^{+\infty} \mathrm{d} x\left[\ln \left(\frac{x}{r_{0}}\right)+H_{k-2}\right] \int_{1}^{+\infty} \mathrm{d} y V_{m}(y)(y+1)^{k-1} F^{(k-2)}[t-r-x(y+1)] .
$$

The same treatment is applied to the second term $A_{2}$ but the computation is longer, as some boundary terms arise. We find that

$$
\begin{aligned}
A_{2} & =-\frac{(-)^{k}}{2(k-2) !} \frac{1}{r} \int_{r}^{+\infty} \mathrm{d} x\left[\ln \left(\frac{x}{r_{0}}\right)+H_{k-2}\right] \int_{1}^{+\infty} \mathrm{d} y V_{m}(y)(y+1)^{k-1} F^{(k-2)}[t+r-x(y+1)] \\
& -\frac{(-)^{k}}{2(k-2) !} \frac{1}{r} \int_{0}^{r} \mathrm{~d} x\left[\ln \left(\frac{x}{r_{0}}\right)+H_{k-2}\right] \int_{1}^{+\infty} \mathrm{d} y V_{m}(y)(y-1)^{k-1} F^{(k-2)}[t-r-x(y-1)] \\
& +\frac{(-)^{k}}{(k-2) !} \frac{1}{r}\left[\ln \left(\frac{r}{r_{0}}\right)+H_{k-2}\right] \int_{1}^{+\infty} \mathrm{d} y V_{m}(y) \varphi_{k-2}(y) F^{(k-3)}(t-r y) \\
& -\sum_{i=1}^{k-3} \frac{(-)^{i}}{(k-2) \cdots(k-2-i)} \frac{1}{r^{k-i-1}} \int_{1}^{+\infty} \mathrm{d} y V_{m}(y) \varphi_{i}(y) F^{(i-1)}(t-r y),
\end{aligned}
$$

where $\varphi_{i}(y)=\frac{1}{2}\left[(y+1)^{i}-(y-1)^{i}\right]$. Then, we make the change of variable $t-r s=$ $t-r-x(y+1)$ in (A15), as well as the changes $t-r s=t+r-x(y+1)$ in the first line of (A16) and $t-r s=t-r-x(y-1)$ in the second line of (A16). Finally, with these new variables, after exchanging the integrations, simplifications occur, yielding the formula (3.13) in the text.

[1] B. Abbott et al. (LIGO Scientific Collaboration and VIRGO Collaboration), Phys. Rev. Lett. 116, 061102 (2016), arXiv:1602.03837 [gr-qc].

[2] B. Abbott et al. (LIGO Scientific Collaboration and VIRGO Collaboration), Phys. Rev. Lett. 116, 241103 (2016), arXiv:1606.04855 [gr-qc].

[3] C. Cutler, T. Apostolatos, L. Bildsten, L. Finn, E. Flanagan, D. Kennefick, D. Markovic, A. Ori, E. Poisson, G. Sussman, et al., Phys. Rev. Lett. 70, 2984 (1993).

[4] C. Cutler and E. Flanagan, Phys. Rev. D 49, 2658 (1994).

[5] L. Blanchet, Living Rev. Rel. 17, 2 (2014), arXiv:1310.1528 [gr-qc].

[6] A. Buonanno and B. Sathyaprakash, in General Relativity and Gravitation: A Centennial Perspective, edited by A. Ashtekar, B. Berger, J. Isenberg, and M. MacCallum (2015), p. 513, arXiv:1410.7832 [gr-qc]. 
[7] L. Blanchet, T. Damour, B. R. Iyer, C. M. Will, and A. G. Wiseman, Phys. Rev. Lett. 74, 3515 (1995), gr-qc/9501027.

[8] L. Blanchet, Class. Quant. Grav. 15, 113 (1998), gr-qc/9710038.

[9] L. Blanchet, G. Faye, B. R. Iyer, and B. Joguet, Phys. Rev. D 65, 061501(R) (2002), erratum Phys. Rev. D, 71:129902(E), 2005, gr-qc/0105099.

[10] L. Blanchet, T. Damour, G. Esposito-Farèse, and B. R. Iyer, Phys. Rev. Lett. 93, 091101 (2004), gr-qc/0406012.

[11] L. Blanchet, B. R. Iyer, C. M. Will, and A. G. Wiseman, Class. Quant. Grav. 13, 575 (1996), gr-qc/9602024.

[12] K. Arun, L. Blanchet, B. R. Iyer, and M. S. Qusailah, Class. Quant. Grav. 21, 3771 (2004), erratum Class. Quant. Grav., 22:3115, 2005, gr-qc/0404185.

[13] L. Blanchet, G. Faye, B. R. Iyer, and S. Sinha, Class. Quant. Grav. 25, 165003 (2008), arXiv:0802.1249 [gr-qc].

[14] G. Faye, S. Marsat, L. Blanchet, and B. R. Iyer, Class. Quant. Grav. 29, 175004 (2012), arXiv:1204.1043 [gr-qc].

[15] G. Faye, L. Blanchet, and B. R. Iyer, Class. Quant. Grav. 32, 045016 (2015), arXiv:1409.3546 [gr-qc].

[16] S. Foffa and R. Sturani, Phys. Rev. D 87, 064011 (2012), arXiv:1206.7087 [gr-qc].

[17] P. Jaranowski and G. Schäfer, Phys. Rev. D 86, 061503(R) (2012), arXiv:1207.5448 [gr-qc].

[18] P. Jaranowski and G. Schäfer, Phys. Rev. D 87, 081503(R) (2013), arXiv:1303.3225 [gr-qc].

[19] T. Damour, P. Jaranowski, and G. Schäfer, Phys. Rev. D 89, 064058 (2014), arXiv:1401.4548 [gr-qc].

[20] L. Bernard, L. Blanchet, A. Bohé, G. Faye, and S. Marsat, Phys. Rev. D 93, 084037 (2016), arXiv:1512.02876 [gr-qc].

[21] L. Bernard, L. Blanchet, A. Bohé, G. Faye, and S. Marsat, Energy and periastron advance of compact binaries on circular orbits at the fourth post-newtonian order (2015), arXiv:1610.07934 [gr-qc].

[22] P. Jaranowski and G. Schäfer, Phys. Rev. D 57, 7274 (1998), gr-qc/9712075.

[23] P. Jaranowski and G. Schäfer, Phys. Rev. D 60, 124003 (1999), gr-qc/9906092.

[24] T. Damour, P. Jaranowski, and G. Schäfer, Phys. Rev. D 62, 021501(R) (2000), erratum Phys. Rev. D 63, 029903(E) (2000), gr-qc/0003051.

[25] T. Damour, P. Jaranowski, and G. Schäfer, Phys. Lett. B 513, 147 (2001), gr-qc/0105038.

[26] L. Blanchet and G. Faye, Phys. Rev. D 63, 062005 (2001), gr-qc/0007051.

[27] V. de Andrade, L. Blanchet, and G. Faye, Class. Quant. Grav. 18, 753 (2001), gr-qc/0011063.

[28] L. Blanchet and B. R. Iyer, Class. Quant. Grav. 20, 755 (2003), gr-qc/0209089.

[29] L. Blanchet, T. Damour, and G. Esposito-Farèse, Phys. Rev. D 69, 124007 (2004), grqc/0311052.

[30] Y. Itoh and T. Futamase, Phys. Rev. D 68, 121501(R) (2003), gr-qc/0310028.

[31] Y. Itoh, Phys. Rev. D 69, 064018 (2004), gr-qc/0310029.

[32] S. Foffa and R. Sturani, Phys. Rev. D 84, 044031 (2011), arXiv:1104.1122 [gr-qc].

[33] W. Bonnor, Philos. Trans. R. Soc. London, Ser. A 251, 233 (1959).

[34] W. Bonnor and M. Rotenberg, Proc. R. Soc. London, Ser. A 289, 247 (1966).

[35] K. Thorne, Rev. Mod. Phys. 52, 299 (1980).

[36] L. Blanchet and T. Damour, Phys. Rev. D 37, 1410 (1988).

[37] L. Blanchet and T. Damour, Phys. Rev. D 46, 4304 (1992).

[38] L. Blanchet and T. Damour, Phil. Trans. Roy. Soc. Lond. A 320, 379 (1986). 
[39] L. Blanchet, Class. Quant. Grav. 15, 89 (1998), gr-qc/9710037.

[40] E. Poisson, Phys. Rev. D 47, 1497 (1993).

[41] H. Tagoshi and T. Nakamura, Phys. Rev. D 49, 4016 (1994).

[42] M. Sasaki, Prog. Theor. Phys. 92, 17 (1994).

[43] H. Tagoshi and M. Sasaki, Prog. Theor. Phys. 92, 745 (1994), gr-qc/9405062.

[44] T. Tanaka, H. Tagoshi, and M. Sasaki, Prog. Theor. Phys. 96, 1087 (1996), gr-qc/9701050.

[45] S. Mano, H. Susuki, and E. Takasugi, Prog. Theor. Phys. 95, 1079 (1996), gr-qc/9603020.

[46] S. Mano, H. Susuki, and E. Takasugi, Prog. Theor. Phys. 96, 549 (1996), gr-qc/9605057.

[47] S. Mano and E. Takasugi, Prog. Theor. Phys. 97, 213 (1997), gr-qc/9611014.

[48] R. Fujita, Prog. Theor. Phys. 127, 583 (2012), arXiv:1104.5615 [gr-qc].

[49] R. Fujita, Prog. Theor. Phys. 128, 971 (2012), arXiv:1211:5535 [gr-qc].

[50] L. Blanchet, Ph.D. thesis, Université Paris VI, Paris (1990).

[51] D. Christodoulou, Phys. Rev. Lett. 67, 1486 (1991).

[52] A. Wiseman and C. Will, Phys. Rev. D 44, R2945 (1991).

[53] K. Thorne, Phys. Rev. D 45, 520 (1992).

[54] M. Favata, Phys. Rev. D 80, 024002 (2009), arXiv:0812.0069 [gr-qc].

[55] M. Favata, Phys. Rev. D 84, 124013 (2011), arXiv:1108.3121 [gr-qc].

[56] I. Gradshteyn and I. Ryzhik, Table of Integrals, Series and Products (Academic Press, 1980).

[57] J. M. Martín-García, A. García-Parrado, A. Stecchina, B. Wardell, C. Pitrou, D. Brizuela, D. Yllanes, G. Faye, L. Stein, R. Portugal, et al., xAct: Efficient tensor computer algebra for Mathematica (GPL 2002-2012), http://www.xact.es/.

[58] T. Damour, B. Iyer, and A. Nagar, Phys. Rev. D 79, 064004 (2009), arXiv:0811.2069 [gr-qc].

[59] L. Blanchet, G. Faye, and B. Whiting, Phys. Rev. D 89, 064026 (2014), arXiv:1312.2975 [gr-qc]. 